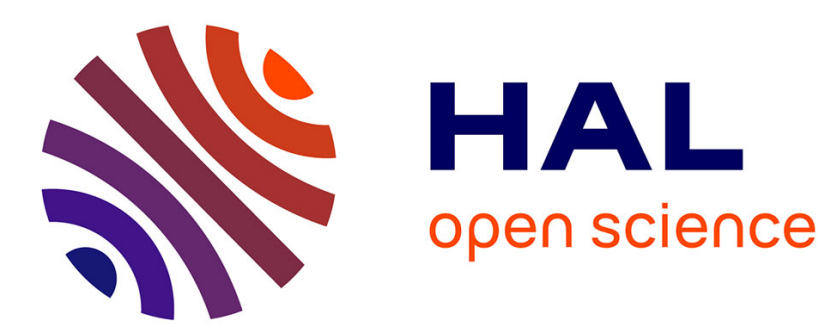

\title{
Design of lightweight airborne MMW radar for DEM generation. Simulation results
}

\author{
R. Rouveure, T. Tourrette, Marion Jaud, P. Faure, M.O. Monod
}

\section{To cite this version:}

R. Rouveure, T. Tourrette, Marion Jaud, P. Faure, M.O. Monod. Design of lightweight airborne MMW radar for DEM generation. Simulation results. Robotics and Autonomous Systems, 2017, 96, pp.26-40. 10.1016/j.robot.2017.07.002 . hal-01761507

\section{HAL Id: hal-01761507 \\ https://hal.science/hal-01761507}

Submitted on 9 Apr 2018

HAL is a multi-disciplinary open access archive for the deposit and dissemination of scientific research documents, whether they are published or not. The documents may come from teaching and research institutions in France or abroad, or from public or private research centers.
L'archive ouverte pluridisciplinaire HAL, est destinée au dépôt et à la diffusion de documents scientifiques de niveau recherche, publiés ou non, émanant des établissements d'enseignement et de recherche français ou étrangers, des laboratoires publics ou privés. 


\title{
Design of lightweight airborne MMW radar for DEM generation. Simulation results.
}

\author{
Raphäl Rouveure ${ }^{1}$, Thibault Tourrette ${ }^{1}$, Marion Jaud ${ }^{2}$, Patrice Faure ${ }^{1}$, Marie-Odile Monod ${ }^{1}$ \\ ${ }^{1}$ National Research Institute of Science and Technology for Environment and Agriculture (IRSTEA), \\ Technologies and Information Systems Research Unit (TSCF), Clermont-Ferrand Regional Center, 9 \\ avenue Blaise Pascal, CS 20085, 63178 Aubière, France \\ ${ }^{2}$ European Institute for Marine Studies (IUEM), Oceanic Domains Laboratory, 29280 Plouzané, \\ France
}

Email addresses: raphael.rouveure@irstea.fr; thibault.tourrette@irstea.fr; marion.jaud@univ-brest.fr; patrice.faure@irstea.fr; marie-odile.monod@irstea.fr

Correspondence should be addressed to Raphaël Rouveure, raphael.rouveure@irstea.fr

\begin{abstract}
There is a growing need for lightweight airborne platforms that could provide precise information about the environment (topography, presence of obstacles, etc.) filling the data gap between aerial/satellite remote sensing and terrestrial systems. A major limitation of classical sensors such as vision or laser is that they are ineffective in degraded visual conditions. Millimeter-wave radar provides an alternative solution to overcome the shortcomings of optical solutions, because in the microwave range, data can be acquired independently of atmospheric conditions and time of the day. The intended application of a new radar sensor is the construction of digital elevation models of the overflown environments. As the design of new radar sensors for light airborne platforms is subject to specific technological constraints, a simulator of airborne radar surveys is developed. The objective of the simulator is to help the designer in defining the main parameters of the future airborne radar, and in developing radar signal processing algorithms.
\end{abstract}

\section{Introduction}

In many situations, aerial or satellite remote sensing can be the only solution to obtain observations about the Earth's surface. Numerous sources of aerial or satellite imagery are widely available online today, free of charge or for purchase depending on the desired spatial resolution of the images. But they cannot systematically address the problem to solve in a project, considering the specific area of study, the desired spatial resolution, the necessity to obtain time series data, etc. Considering these elements, a low-cost and high-resolution perception system based on Unmanned Aerial Vehicle (UAV) can provide alternative data sources, with resolution performances and implementation capacities complementary to current satellites/airplanes and ground surveying systems.

It is now apparent that the growth potential of the UAV sector is important. The Association for Unmanned Vehicle Systems International (AUVSI) in a report of March 2013 [1] forecasts an explosion of the UAV market, particularly in the areas of precision agriculture (remote sensing and precision application) and of public safety (public protection from natural or man-made disasters, involving the intervention of emergency services in crisis situations). This report concludes that "the economic impact of the integration of UAS (Unmanned Aircraft Systems) into the NAS (United States National Airspace System) will total more than $\$ 13.6$ billion" and "will create more than 70,000 new jobs in the first three years" of integration. In France, the report of April 2015 published by IDATE Research [2] confirms this trend, with a global market for civil drones estimated at about $\$ 10.8$ billion 
by 2020. In the more specific domain of environmental monitoring, UAV technology could represent a technological break for data acquisition: in a report of May 2013, the United Nations Environment Programme (UNEP) outlines that UAV can provide "a low-cost and low-impact solution to environmental managers working in a variety of ecosystems" [3]. In this report, UAV used within the framework of these applications are called "eco-drones" or "conservation drones".

Ease of use and perceptual capabilities make UAVs interesting for the monitoring of the environment or of natural disasters. However, a certain number of scientific and technological difficulties persist. One of these difficulties is related to the increased autonomy of UAVs. An increased autonomy implies that the UAVs can face any degraded visual environments (DVE) for perception and navigation purposes. DVE refers to circumstances wherein optical perception systems (vision, laser) are ineffective due to weather conditions (rain, fog, etc.) or the presence of obscurants (dust, smoke). In such situations, millimeter-wave (MMW) radar can provide an alternative solution to overcome the limitations of optical sensors. Indeed, it is no longer necessary to demonstrate the efficiency of microwave technology for perception in outdoor environments. Due to a millimeter or centimeter wavelength, MMW radars are robust sensors in degraded visual conditions [4],[5],[6]. In remote sensing applications, radars have been initially designed for large platforms such as airplanes or satellites. With the development of UAV-based applications, these systems are progressively adapted for smaller platforms in terms of dimension, weight, energy consumption and cost [7],[8],[9]. Our objective is to develop a radar system for light airborne platforms, in order to build digital elevation models (DEM) of the overflown environments independently of visual conditions and time of the day. Applications being considered are related to all-weather perception: monitoring of natural areas, DEM construction and obstacle detection for crisis intervention, UAV autonomous navigation, etc. As a first step of the radar sensor design, a simulator of airborne radar is developed in order to help to define the best radar configuration and to develop radar signal processing algorithms.

The radar simulator is described in the paper. Section 2 introduces preliminary results obtained in 2D map construction with MMW radar developed at Irstea Institute for autonomous ground vehicle applications. Principle of DEM construction with a MMW radar altimeter is also presented. The main components of the simulator are described more in details in Section 3: trajectory modeling, radar modeling and environment modeling. Radar signal processing is developed in Section 4, and examples of DEM construction obtained with the simulator are given in Section 5. Section 6 concludes the paper.

\section{From 2D Mapping to 3D Mapping with MMW radar}

\subsection{Preliminary Results in Perception with K-band PELICAN Radar}

PELICAN radar has been developed at Irstea Institute for perception and mapping applications in the domain of Autonomous Ground Vehicle (AGV) and environmental monitoring [10],[11],[12]. PELICAN is a Frequency Modulated Continuous Wave (FMCW) radar. It is using a $\mathrm{K}$ band $(24 \mathrm{GHz})$ carrier frequency. It is equipped with a planar patch array antenna, which rotates in the horizontal plane (rotational speed: $60 \mathrm{rpm}$ ) in order to obtain panoramic views of the environment in the range 5-100 meters. With small size (length $26 \mathrm{~cm}$, width $24 \mathrm{~cm}$, height $30 \mathrm{~cm})$ and light weight $(<10 \mathrm{~kg})$, PELICAN can be positioned on various vehicles including robots and small boats. A general view of PELICAN radar and its implementation on an experimental vehicle are presented in the upper left and lower left corners of Figure 1(a). The antenna produces a fan beam radiation pattern: the main beam has a narrow beamwidh in the horizontal dimension (azimuth), and a wider beamwidth in the vertical dimension (elevation). 
PELICAN radar is associated with the R-SLAM algorithm which is based on Simultaneous Localization And Mapping (SLAM) principles [13],[14],[15]. R-SLAM algorithm merges the successive panoramic radar images, with the objective of constructing 2D map of the environment and computing the trajectory within the map. An example of map construction in semi-urban environment (baseball stadium, Aubière, France; localization: $45^{\circ} 45^{\prime} 32.00^{\prime \prime} \mathrm{N}, 3^{\circ} 06^{\prime} 28.00^{\prime \prime} \mathrm{E}$ ) obtained with PELICAN radar and R-SLAM algorithm is presented in Figure 1. Figure 1(a) is the aerial view of the test zone extracted from Google Earth. The red dots show the trajectory followed by the experimental vehicle (GPS data). Marks $A$ and $B$ indicate departure and arrival points respectively. In this example, 300 panoramic radar images are used to produce the global map presented in Figure 1(b). Color levels refer to the amplitudes of the reflected radar signals. The cyan dots indicate the computed trajectory.

One can notice that the use of a fan beam antenna constitutes a main characteristic and a major limitation of PELICAN radar. On one hand, the radiation pattern makes the radar robust to some severe positioning variations in pitch and roll of the robot when it navigates in non-flat environments. On the other hand, the altitudes/heights of the targets are not measured and PELICAN radar can only be used to build 2D representation of the environment. So an alternative approach must be followed in order to be able to take into account and represent $3 \mathrm{D}$ environments with radar measurements.

\subsection{D Mapping Principle with Radar}

In aerial and satellite remote sensing domains, radar imagers have been used for a long time to build 3D representations of the environment. Several approaches have been developed, such as radarclinometry, the use of polarimetry, interferometry or radargrammetry. Radarclinometry [16] requires the use of complex backscatter models, making its use difficult in inhomogeneous areas. Polarimetry [17] complicates the radar architecture because it implies the measurements of several polarimetric modes ( $\mathrm{HH}, \mathrm{HV}, \mathrm{VV}, \mathrm{VH})$. Interferometry (InSAR) [18] is based on phase difference measurements between two SAR images. It implies a correct correlation between both images, which can be difficult with areas covered by vegetation. Radargrammetry [19] is the equivalent of photogrammetry in the optical domain. Due to the inherent speckle effect present in radar images, the matching between the homologous points of the radar images can be complex.

In remote sensing domain, radar altimeters can also be used to recover $3 \mathrm{D}$ information about the overflown environment. Indeed, satellite or airborne altimeters use the ranging capabilities of radar sensors to measure surface topography. Major applications of radar altimetry are related to ocean and ice studies [20],[21]. Radar altimeters are designed for operation in either beam-limited or pulselimited mode. In beam-limited mode, the objective is to obtain the smallest possible radar footprint, which can be difficult considering the radar altitude and the corresponding required antenna size (the antenna aperture, which defines the radar footprint, is inversely proportional to the antenna size). In pulse-limited mode, which is used by a majority of spaceborne radar altimeters, a broad antenna beam is used, requiring a smaller antenna. When the radar pulse intersects the ground or the sea, it illuminates a growing disk which spreads out across the beam-limited footprint: the temporal evolution of the reflected radar pulse is interpreted in order to estimate the distance between the radar altimeter and the reflecting surface. Surface irregularities such as significant wave height can also be estimated with this approach. 
(a)
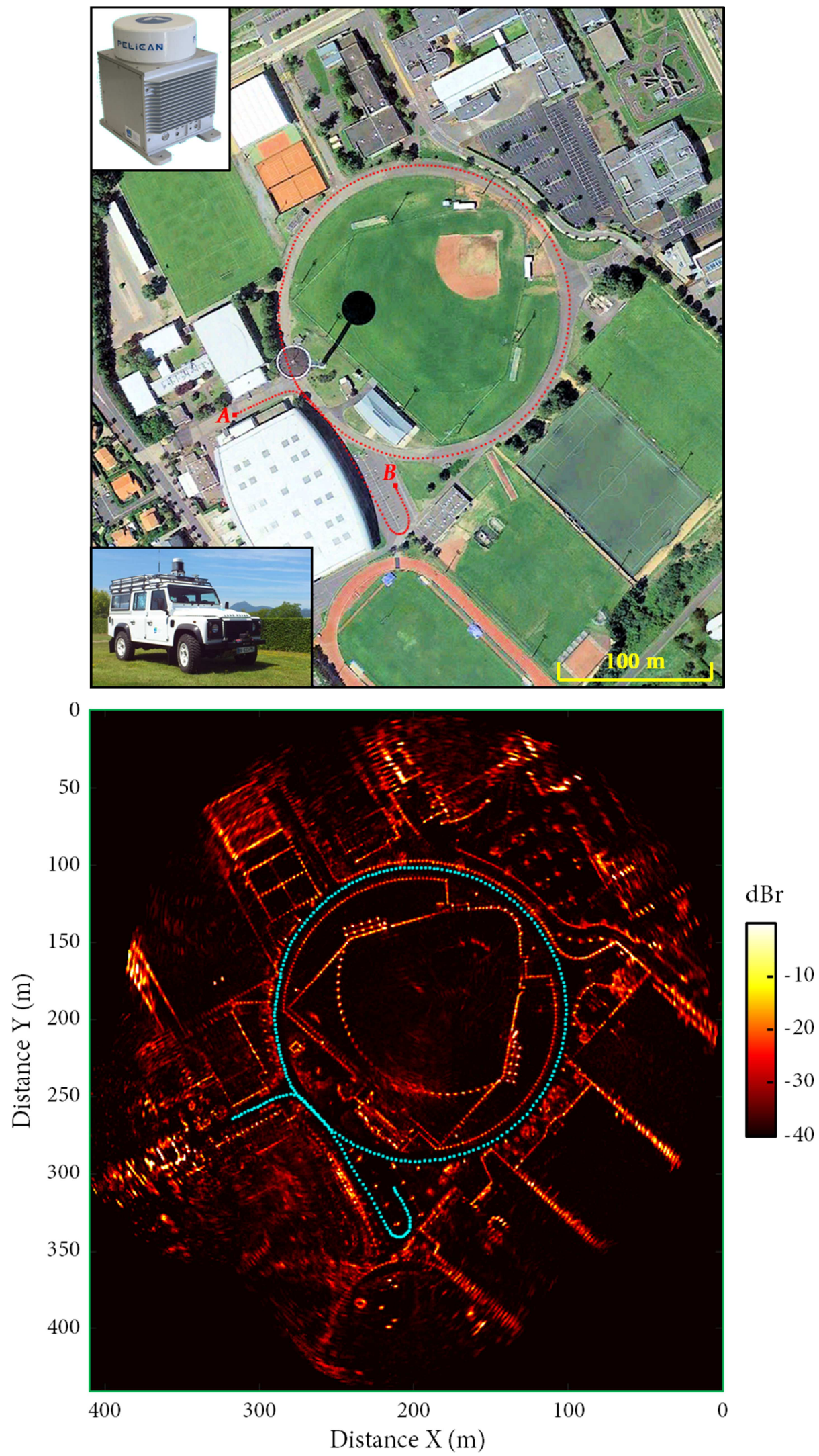

FIGURE 1: 2D radar map obtained with PELICAN radar and R-SLAM algorithm. (a) RGB aerial image of the test zone, extracted from Goggle Earth data. Upper left image: view of PELICAN radar. Lower-left image: experimental vehicle. The red dots show the GPS trajectory. (b) 2D radar map. The cyan dots indicate the computed radar trajectory. 
To solve the problem of helicopter landing in DVE, several "see-through" solutions are under development. These solutions are mainly based on the use of 3D beam-limited radar altimeters [22],[23]. These radars are using pencil beam antennas, in order to produce real-time 3D synthetic images of the ground and proximate surface hazards in and around the landing zone. The Brownout Landing Aid System Technology (BLAST) presented in [22] uses a $94 \mathrm{GHz}$ MMW radar, which is adapted from a radar missile seeker: its cost remains incompatible with UAV-based low-cost applications. Similar approaches can be found in the mobile robotics domain for 3D perception of the environment [24],[25].

The proposed solution is based on the use of a beam-limited radar altimeter. Radar equipped with a pencil beam antenna is positioned on the UAV. The narrow beam pattern of the antenna, combined with the low altitude of the UAV, produces a size-limited footprint on the ground allowing a precise localization of the radar-ground measurement. The antenna is mechanically scanned in order to cover an area ahead of the platform (electronic scanning remains too expensive and too complex for UAV applications). To reduce the scanning complexity, a "whisk broom scanner" solution is adopted. The antenna rotation covers transversal scan (scanning angle $\beta$ ), so that a strip of ground perpendicular to the UAV trajectory is illuminated. The longitudinal scan is obtained with the displacement of the UAV. An illustration of this approach is presented in Figure 2.

The incidence angle $\alpha$ is maintained constant, but it can be adjusted depending on the altitude and/or the velocity of the UAV. Radar distance measurements are combined with the $6 \mathrm{D}$ localization of the UAV in order to produce a synthetic 3D image of the overflown environment.

The choice of a pencil beam solution is guided by several factors:

- direct access to radar-target distances, with low geometrical distortions (layover and foreshortening) by comparison with SAR imaging systems [26]. In a SAR imaging systems, 3D elements are projected in a two-dimensional slant image plane: the reconstruction of 3D information with single SAR images is thus mainly limited to building height estimation [27],[28].

- relative simplicity of data processing by comparison with SAR and inSAR systems. Real-time data processing can be reasonably expected, as well as the use of a "reasonable" onboard computer in terms of size, weight, consumption and cost.

- simplicity of the microwave architecture by comparison with inSAR systems, with a gain in size, weight and cost

Numerous radar parameters have to be defined: intrinsic parameters such as antenna aperture, antenna scanning velocity or characteristics of the transmitted radar signal; and extrinsic parameters such as UAV altitude and velocity. In order to better define and to better control all these parameters, a simulation phase has been decided. The simulator is developed with an "engineering oriented" approach, and the objectives are to assist the designer in defining the most suitable radar configuration, and to develop processing algorithms (radar signal processing, 3D reconstruction algorithms, etc.) with the simulation of radar signals. 


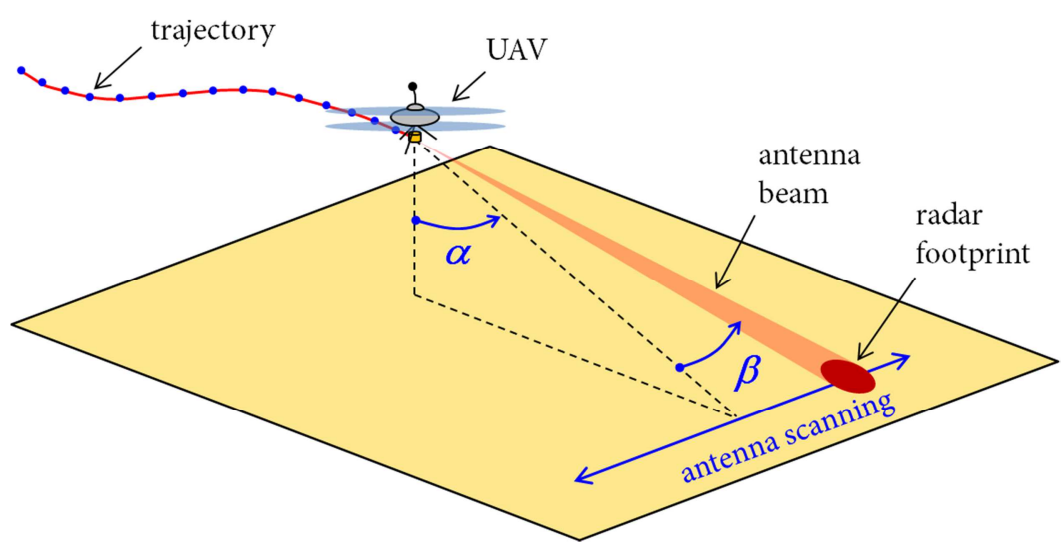

FIGURE 2: Principle of 3D mapping with a radar altimeter. A "whisk broom scanner" solution is adopted in order to reduce the complexity of the scanning process.

\section{Description of the Simulator}

The simulator is based on the following structure (see Figure 3):

(i) A geometrical model of the UAV's trajectory, in terms of position, attitude and velocity,

(ii) A model of MMW radar, in order to describe the radar antenna and the backscattered radar signal,

(iii) A model of the overflown environment, described with geometric and electromagnetic properties.

Once the models are configured (i.e. a virtual environment is described, a trajectory is defined and radar parameters are adjusted), a radar survey is simulated. Based on radar distance measurements and $6 \mathrm{D}$ radar localization, a DEM is computed and compared to the model of the environment. Different test scenarios (trajectory, environment) are used in order to estimate the optimal radar configuration.

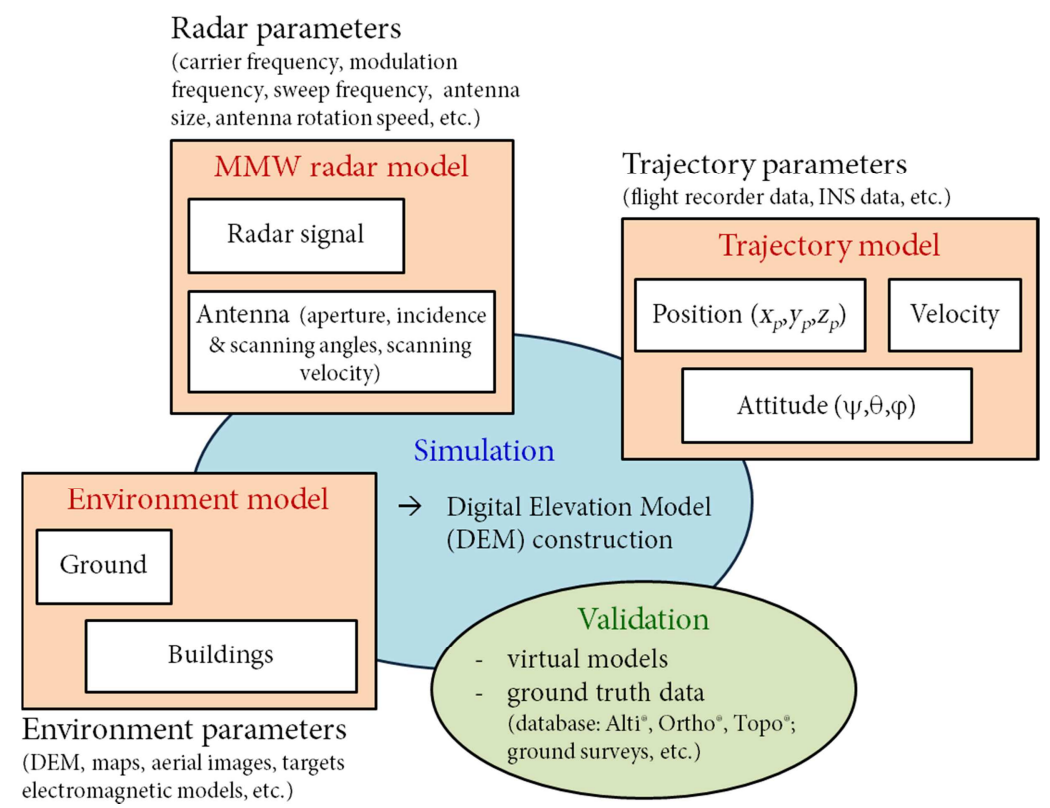

FIGURE 3: General structure of the airborne radar simulator. Trajectory and environment models are used to define various test scenarios. The computed DEM are compared with the models of the environments in order to determine the optimal radar configuration.

The objective is not to build complex models of the environment, taking into account all aspects of the interactions between the incident wave and the surface irregularities. Our goal is to generate realistic enough radar signals, taking into account size and orientation of the radar beam, movements 
of the UAV, variations of backscattered energy, etc., in order to define radar parameters, and to develop signal processing algorithms without having real radar data.

When accurate simulations are required, full-wave electromagnetic methods (EM) such as finitedifference time domain (FDTD) methods are recommended [29],[30]. But these methods impose high memory requirements, long calculation times, and high computing power. In that sense, they are not adapted for the description of large environments. We have selected a ray tracing approach, which is well suited for signal processing development and validation [31],[32],[33],[34]. In this approach, the environment is modeled with facets of known geometrical and electromagnetic properties. The radar signal is then computed in the time domain by simulating the reflections over the facets, considering that the radar wave acts like an optical wave. In can be noticed original solutions that combine several approaches: in [31], a full-wave electromagnetic method is used for the description of specific targets, and is associated with a ray tracing method for the description of the large environments in which the specific targets are positioned.

\subsection{UAV Trajectory Modeling}

The different phases of flight can be modeled, either takeoff, climb, cruise, descent or landing. The trajectory is either virtual, or based on real data collected by a flight recorder. The objective of the trajectory modeling module is to simulate data provided by the Inertial Navigation System (INS) that will be used on the airborne platform.

The flight of the UAV is simulated considering the following parameters: (i) the successive 3D positions $\left(x_{p}, y_{p}, Z_{p}\right)$; (ii) the UAV attitude; and (iii) the speed of the UAV. The vehicle-carried vertical axis system $\left(X_{v}, y_{v}, Z_{v}\right)$ is obtained by a translation of an earth-fixed reference frame. It has its origin at the center of gravity of the UAV. The $x_{V}$ axis is directed north, the $y_{V}$ axis east, and the $Z_{V}$ axis down. The origin of the body axis system $\left(x_{b}, y_{b}, z_{b}\right)$ is also the vehicle center of gravity. The $x_{b}$ axis is directed toward the nose of the UAV, the $y_{b}$ axis toward the right, and the $z_{b}$ axis toward the bottom of the UAV (see Figure 4).

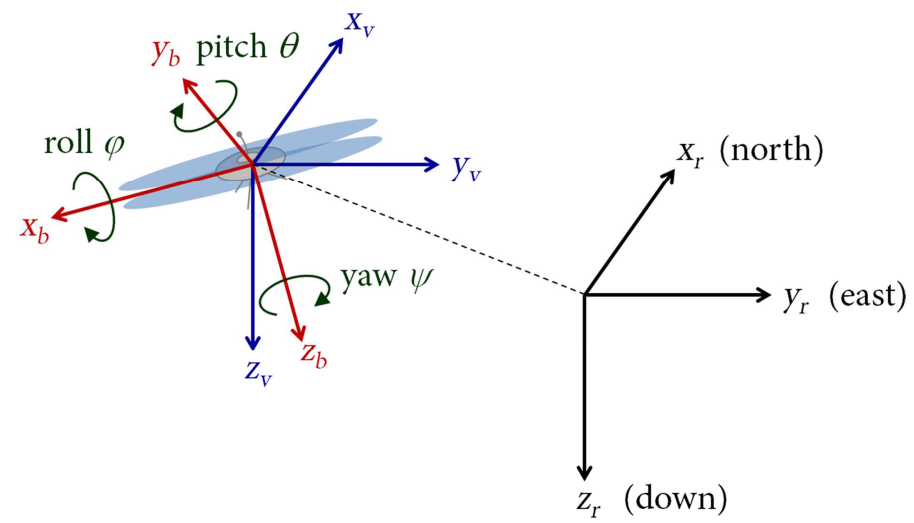

FIgURE 4: Definition of the axis systems and of the Euler angles. $\left(x_{r}, y_{r}, Z_{r}\right)$ is the earth-fixed reference frame, $\left(x_{v}, y_{v}, Z_{v}\right)$ the vehicle-carried vertical axis system, and $\left(x_{b}, y_{b}, Z_{b}\right)$ the body axis system. The Euler angles $\varphi, \theta$ and $\psi$ define three rotations required to transform the vehicle-carried vertical axis system $\left(x_{v}, y_{v}, Z_{v}\right)$ to the body axis system $\left(x_{b}, y_{b}, Z_{b}\right)$.

The UAV 3-dimensional attitude is defined by Euler angles: roll $\varphi$, pitch $\theta$, yaw or heading $\psi$. The Euler angles define three rotations required to transform the vehicle-carried vertical axis system $\left(x_{b}, y_{n}, Z_{v}\right)$ to the body axis system $\left(x_{b}, y_{b}, z_{b}\right)$. These three rotations are used to project the radar footprint into the earth fixed reference frame. The accuracy of the inertial navigation system measuring $(\varphi, \theta, \psi)$ will impact the accuracy of the radar beam footprint positioning, and therefore the accuracy of the $3 \mathrm{D}$ reconstruction. 


\subsection{MMW Radar Modeling}

Radar modeling and radar developments within this project are based on Frequency Modulation Continuous Wave (FMCW) principles. This technology is known and used for several decades [37],[11]. FMCW radars are developed and used at Irstea Institute within the framework of AGV applications. FMCW radars are well matched for short and medium range distance applications, because they eliminate the blind zone near the radars (in the case of pulse radars, the blind zone is introduced by the duration of the transmitted pulse). Due to the coupling between transmitting and receiving stages, the transmitted power and thus the maximum range are limited with FMCW radars. But it is not a constraint in our application considering the envisaged radar-target distances $(\ll 1 \mathrm{~km})$. Moreover, the relative simplicity of FMCW architectures can help to develop small-sized systems, compatible with lightweight UAV.

In FMCW radars, the oscillator transmits a signal of linearly increasing frequency $\Delta f$ over a period $t_{m}$. This signal is transmitted into the air via the antenna. At the receiver stage, a part of the transmitted signal is mixed with the signals received from the $i$ targets present in the field of view of the radar. The signal which appears at the output of the mixer is filtered and amplified in order to isolate the beat signal $s_{b}$. Let us consider $i$ targets located at distance $r_{i}$ from the radar, with radial velocities $V_{r i}$. The transmitted signal is linearly modulated over a period $t_{m}=1 / f_{m}$ with a sawtooth function, with a sweep frequency $\Delta f$ centered about $f_{0}$. In that case, the beat signal $s_{b}$ can be written as [11],[37],[38]:

$$
s_{b}(t)=k \sum_{i} a_{t} a_{r i} \cos (2 \pi \underbrace{\left(2 \Delta f f_{m} \frac{r_{i}}{c}+2 f_{0} \frac{V_{r i}}{c}\right)}_{f_{b i}} t+\Phi_{i}),
$$

where $a_{t}$ is the amplitude of transmitted signal, $a_{r i}$ and $\Phi_{i}$ respectively the amplitude and a phase term of the signal received from target $i$, and $k$ a mixer coefficient. As it can be seen in (1), the beat signal $s_{b}$ is the sum of $i$ frequency components $f_{b i}$, (plus a phase term $\Phi_{i}$ ), each of them corresponding to a particular target $i$ :

$$
f_{b i}=\underbrace{2 \Delta f f_{m} \frac{r_{i}}{c}}_{f_{r}}+\underbrace{2 f_{0} \frac{V_{r i}}{c}}_{f_{d o p}} .
$$

The first part $f_{r}$ of (2) only depends on the range $r_{i}$, and the second part $f_{\text {dop }}$ is the Doppler shift induced by the radial velocity $V_{r i}$ : the measurement of $f_{b i}$ is subject to range-velocity ambiguity. If $V_{r i}=0$, one can see that $f_{b i}$ is proportional to the radar-target distance $r_{i}$.

From (1), we see that the amplitudes of the frequency components of the beat signal are proportional to the term $\left(a_{t} a_{r i}\right)$. Thus, considering that $a_{t}$ is constant, the amplitudes of the frequency components are proportional to the amplitudes $a_{r i}$ of the received signals. The radar equation is an efficient tool to study the parameters that affect $a_{r i}$ The radar equation gives a relationship between the expected received power $p_{r}$ from a target, its radar cross section (RCS) $\sigma$, its range $r$, and intrinsic radar characteristics. The simple form of the radar equation is given by [37]:

$$
p_{r}=\frac{p_{t} G^{2} \lambda^{2} \sigma}{(4 \pi)^{3} r^{4}}
$$


with $p_{t}$ transmitted power, $\lambda$ wavelength and $G$ antenna gain (monostatic case, i.e. the same antenna is used for transition and reception). The RCS $\sigma$, expressed in meter square $\left(\mathrm{m}^{2}\right)$, is a measure of the degree of visibility of the target to the radar i.e. how a target re-radiates the energy of the incident radar signal. $\sigma$ depends on radar characteristics (wavelength, polarization) and on intrinsic parameters of the target: size, surface roughness, nature of constituting materials. It also depends on the orientation of the target to the radar. (3) is valid when considering point target (i.e. radar-target distance 》 target's dimension). In the case of spatially extended targets such as ground or vegetation (the word clutter is commonly used to describe this kind of elements), the term backscatter coefficient $\sigma_{0}$ is introduced: it is the normalized radar cross-section (the average RCS per unit of surface). The cross section $\sigma$ of the clutter can therefore be written as:

$$
\sigma=\sigma_{0} A
$$

with $A$ surface of the illuminated area. Substituting (4) in (3), the power $p_{r}$ received from a distributed target is given by:

$$
p_{r}=\frac{p_{t} G^{2} \lambda^{2} \sigma_{0} A}{(4 \pi)^{3} r^{4}}
$$

A characteristic of a radar sensor in comparison with other technologies such as laser is due to the radar beam which cannot be considered as a point. A rough estimation of the half-power radar beam width $\Theta$ is given by the ratio of the wavelength $\lambda$ to the antenna size $d$ [39]:

$$
\Theta \approx \frac{\lambda}{d}
$$

From (6), one can see that $\Theta$ is inversely proportional to the size $d$ : the higher the size $d$, the smaller the antenna aperture $\Theta$. And for a desired $\Theta$, a smaller $\lambda$ (i.e. a higher carrier frequency $f_{0}$ ) will allow to reduce the size of the antenna. An antenna radiates energy in all directions. The direction of maximum radiation is called main lobe and is defined with two angles: the azimuth angle $\Theta_{a z}$ and the elevation angle $\Theta_{e l .} \Theta_{a z}$ and $\Theta_{e l}$ define the half-power (-3dB) antenna aperture. Away from the main lobe are the side lobes, which correspond to radiation in undesired directions. Side lobes are characterized by the directions of radiation and the side lobe ratio (ratio between the amplitude of the main lobe and the amplitude of the side lobes). For our application, we simulate a narrow beam antenna, characterized by a small antenna aperture in azimuth and elevation. A simulation of phased array radar antenna radiation pattern is presented in Figure 5. This antenna has a half power aperture of $2.0^{\circ}$ in azimuth and elevation planes. Its first side lobe level is about $-20 \mathrm{~dB}$. 
(a)

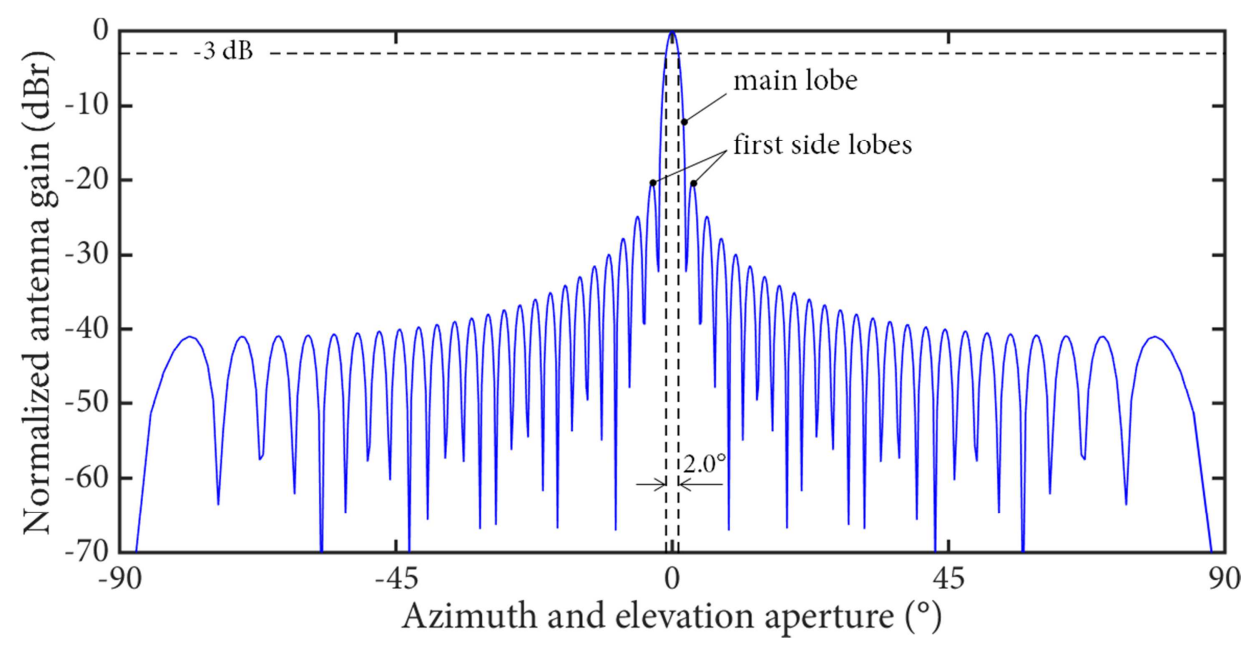

(b)

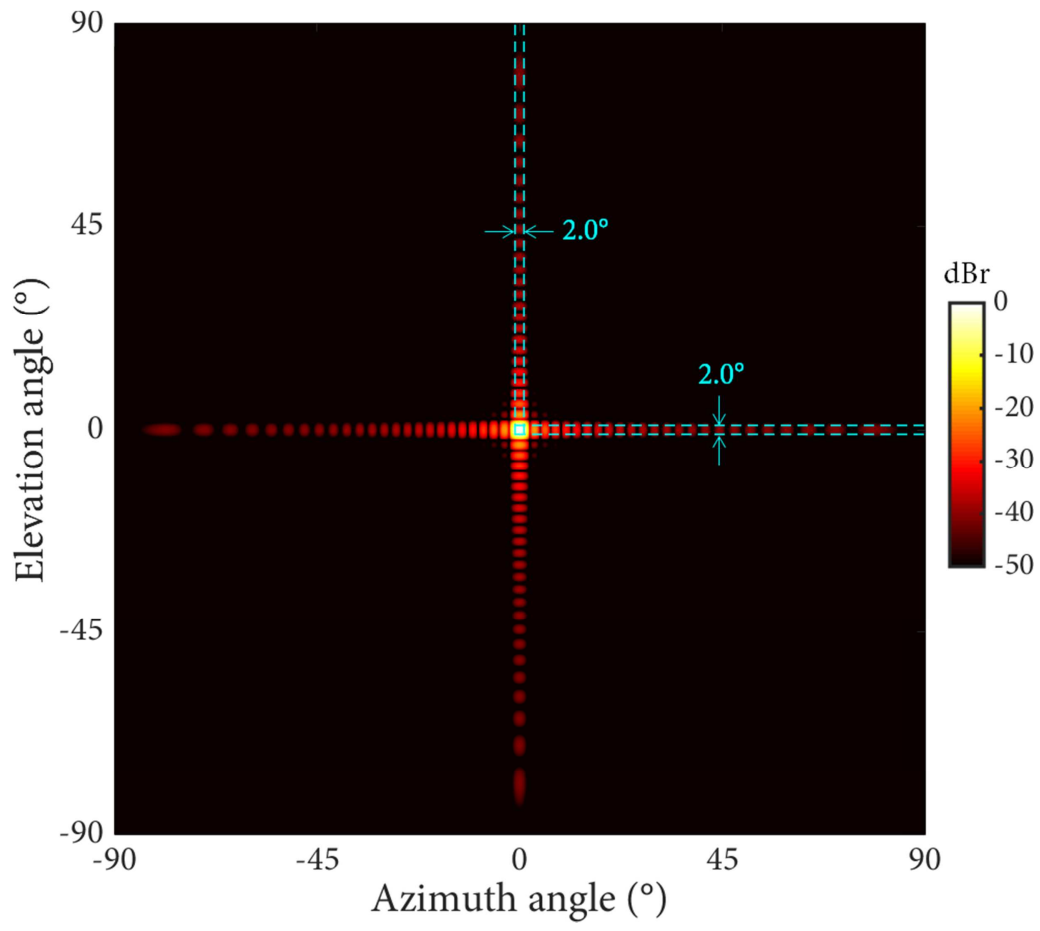

FIGURE 5: Simulation radiation pattern of a pencil beam antenna. The half-power beamwidth is $2.0^{\circ}$ in azimuth and elevation planes. (a) Normalized gain of the antenna along the main azimuth and elevation axis. (b) 3D resulting antenna radiation pattern.

The orientation of the beam is controlled with a mechanical scanning of the antenna. In order to reduce the complexity of this scanning, radar acts like a whisk broom scanner: the antenna scans across the UAV's path, and the 2D scanning is obtained with the displacement of the UAV.

\subsection{Environment Modeling}

The simulator is based on a classical ray tracing approach. This approach is commonly used in radar domain when considering the description of large environments [31],[32],[33],[34].

The environment is described with a set of small facets via a Delaunay triangulation. Each triangle (facet) is considered as an elementary scatterer (reflector), and is characterized by geometrical and electromagnetic properties:

- 3D coordinates of the triangle vertices $\left(p_{1}, p_{2}, p_{3}\right)$ in a reference frame and a normal vector $\vec{n}$ to the surface for the geometrical properties; 
- a backscatter coefficient $\sigma_{0}$ for the electromagnetic properties.

The positions of the triangle vertices allow to compute the distances to the radar and the surface of the triangle. The normal vector $\vec{n}$ is used to determine the local incidence angle $\alpha$. The local incidence angle $\alpha_{l}$ is the angle between the incident radar signal and the normal vector to the surface (see Figure 6).

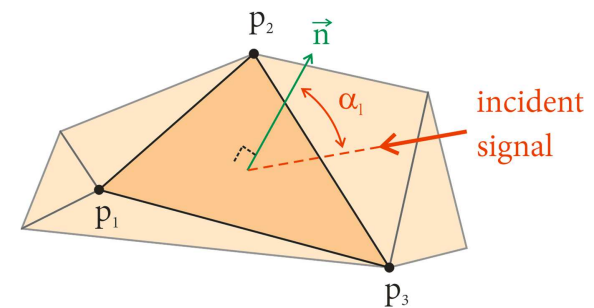

FIGURE 6: Delaunay triangulation of the modeled environment. $\left(p_{1}, p_{2}, p_{3}\right)$ are the vertices of the triangle and $\vec{n}$ the normal vector to the surface of the triangle. $\alpha_{l}$ is the local incidence angle.

Each visible triangle acts like an elementary scatterer, and the measured radar signal is computed in the time domain with the sum of the elementary contributions of all facets located in the radar footprint. The algorithms simulate reflections on the facets, taking into account all possible situations (depending on the sophistication of the developed simulator): single bounce, double-bounce, multibounce, shadowing, etc.

We have considered two major simplifications in our simulator:

- only single-bounce are taken into account. Double-bounce and multi-bounce reflections are not used to compute the reflected radar signal: the energy reflected by a triangle comes only from the radar and not from other triangles. Such a simplification does not allow to build realistic radar image such as those presented for example in [32],[35],[36]. But this simplification is acceptable if we consider the use of a small antenna aperture, and if we consider that the desired simulated data is the distance of the first echo.

- a constant gamma model is used to describe the backscatter coefficient $\sigma_{0}$. It is a simple model of $\sigma_{0}$ but often used in radar remote sensing [40],[41]. In the constant gamma model, $\sigma_{0}$ is expressed as:

$$
\sigma_{0}=\gamma \cos \left(\alpha_{1}\right)
$$

where $\gamma$ is a constant that describes the surface scatter effectiveness. The surface scatter effectiveness is a function of the land cover type and surface roughness. Table 1 gives some values of $\gamma$ for several types of terrain. This model provides correct agreement with most measurements, if considering local incidence angles not too close to $0^{\circ}$ and to $90^{\circ}$. More sophisticated approaches can be used such as in [33], with electromagnetic models based on Kirchhoff s approximation, small perturbation methods, physical optics. Data extracted from databases can also be used, such as the measurement campaigns realized by F.T. Ulaby and M.C Dobson [42].

TABLE 1: Values of the surface scatter effectiveness $\gamma$ for several types of terrain.

\begin{tabular}{cr}
\hline Type of terrain & $\gamma$ \\
\hline City & $83.17 \mathrm{e}^{-3}(-10.8 \mathrm{~dB})$ \\
Mountain & $6.92 \mathrm{e}^{-3}(-21.6 \mathrm{~dB})$ \\
Forest & $3.16 \mathrm{e}^{-3}(-25.0 \mathrm{~dB})$ \\
Rolling hills & $1.32 \mathrm{e}^{-3}(-28.8 \mathrm{~dB})$ \\
Desert & $0.06 \mathrm{e}^{-3}(-42.0 \mathrm{~dB})$ \\
\hline
\end{tabular}


From a geometric standpoint, the environment is modeled with flat or hilly surfaces. Buildings can be added as parallelepiped elements, defining the sizes, locations and orientations (see Figure 7(a)). Data extracted from georeferenced databases can also be used to build more realistic environments. An example with the Vallée de Chaudefour (Auvergne, France) computed from data of the French database BD Alti ${ }^{\circledR}\left(\mathrm{IGN}^{\odot}\right)$ is shown in Figure 7 (b).

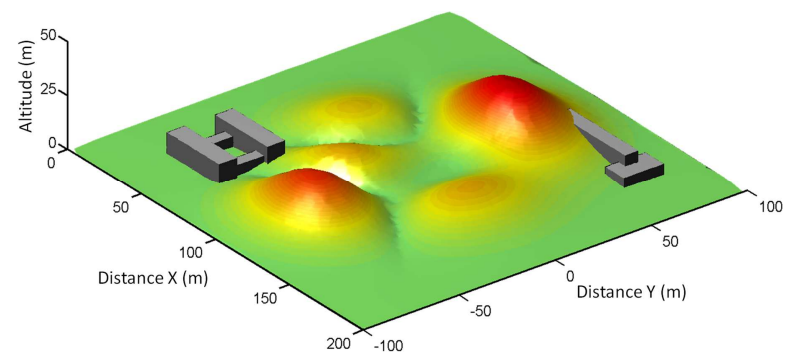

(a)

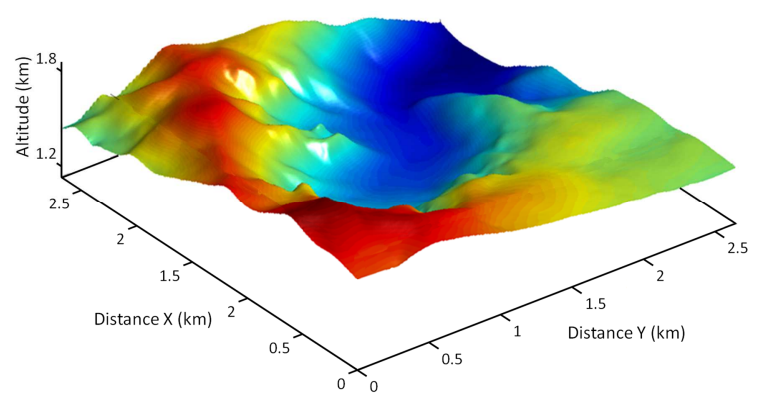

(b)

FIGURE 7: Examples of environment modeling. (a) Virtual environment involving buildings. (b) More realistic environment constructed with the French reference database BD Alti $^{\circledR}\left(\mathrm{IGN}^{\odot}\right)$. The area corresponds to the Vallée de Chaudefour (Auvergne, France).

\section{Radar Signal Processing}

\subsection{Choice of the Carrier Frequency $f_{0}$}

The choice of the carrier frequency $f_{0}$ is not a trivial problem considering its influences on numerous parameters such as maximum range, attenuation through the atmosphere (due to oxygen or water vapor molecules, presence of dust or of rain), radar dimensions, etc. $f_{0}$ is taken into account in the simulator as it can be seen in (1), but the choice of $f_{0}$ is not an expected output of the simulator. Indeed, the final choice of $f_{0}$ is the compromise between several criteria, including intrinsic parameters related to technological aspects and extrinsic parameters related to specific constraints of the expected applications.

Within the framework of our application, three criteria appear predominant. The first one is related to the dimensions of the radar. The final objective is to develop a radar sensor for lightweight UAV, thus the weight and sizes of the radar must be carefully considered. In that sense, the necessity to develop compact radar guides the choice of $f_{0}$ toward high-frequency domain, considering that the dimensions of the microwave components are inversely proportional to the carrier frequency as it can be seen in (6). The second one is related to the cost and the availability of microwave components (oscillator, antenna, etc.), which are still constraints for the development of new devices and applications in the industrial or research domains. It is a fluctuating situation, because due to the emergence of new markets, up-to-date components are regularly proposed by dealers. The last one is related to regulatory constraints. The radiation of RF energy is subject to authorizations which are managed by several organizations, at national and international levels. All these regulation laws lead to the definitions of limitations such as prohibited frequency bands, or bandwidth and power limitations.

The $\mathrm{E}$ band $(60-90 \mathrm{GHz})$ and the $\mathrm{W}$ band $(75-110 \mathrm{GHz})$ appear as a good compromise between expected performance, overall dimensions and weight, availability and cost. With the development of automatic cruise control and anti-collision applications in the automotive sector, $77 \mathrm{GHz}$ components are now available for "low-cost" civilian applications. In the remainder of the paper, the carrier frequency $f_{0}=77 \mathrm{GHz}$ has been selected in order to illustrate the simulation results. 


\subsection{Radar Signal Processing, Target Detection and DEM Construction}

At each computation step of the simulation process, the position $\left(x_{p}, y_{p}, Z_{p}\right)$ and the attitude $(\varphi, \theta, \psi)$ of the UAV are determined from the trajectory. The incidence and scanning angles $(\alpha, \beta)$ are calculated in absolute coordinates. From these data, the position of the radar footprint is determined, and the facets of the environment which are intercepted by the antenna pattern are identified. The process is illustrated in Figure 8. In this example, the antenna intercepts at the same time the top of the building and the ground. Each facet which belongs to the footprint is considered as an elementary scatterer and is used to compute the backscattered signal measured by the radar based on (1).

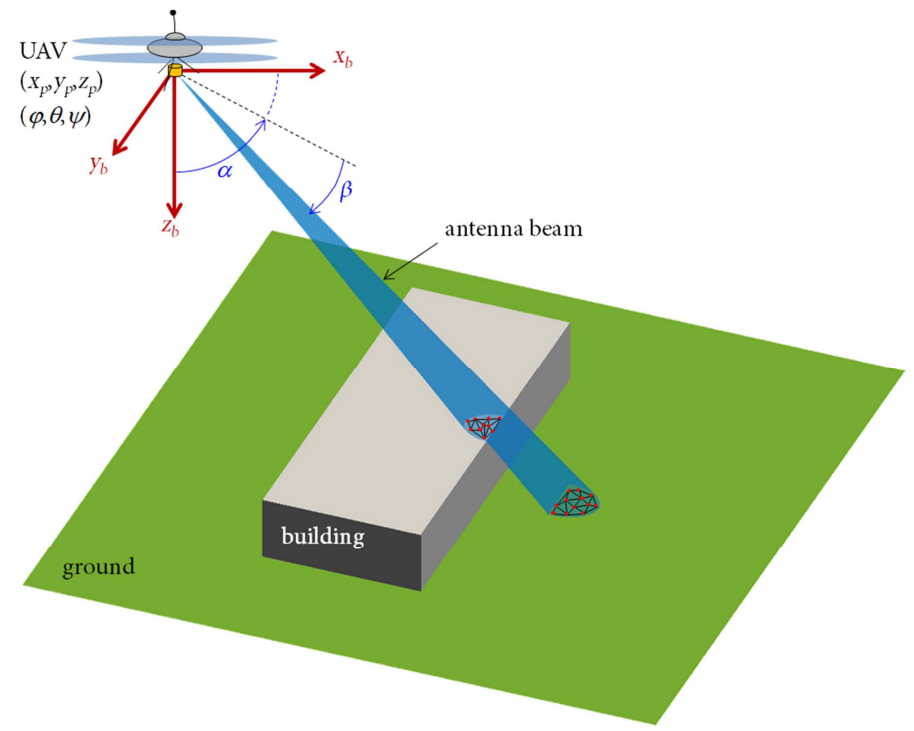

FIGURE 8: Localization of the radar footprint. Based on UAV position $\left(x_{p}, y_{p}, Z_{p}\right)$ and attitude $(\varphi, \theta, \psi)$, and on radar antenna orientation (incidence angle $\alpha$ and scanning angle $\beta$ ), the radar footprint is localized in the environment.

An example of FMCW radar signal processing and target detection is presented in Figure 9. The radar parameters used for this simulation are described in Table 2. The configuration of radar survey is as described in Figure 8: the UAV is $30 \mathrm{~m}$ above the ground, and the antenna pattern intercepts at the same time the top of a building and the ground.

TABLE 2: Parameters of the simulated FMCW radar.

\begin{tabular}{lc}
\hline Carrier frequency $f_{0}$ & $77 \mathrm{GHz}$ \\
Chirp repetition frequency $f_{m}$ & $360 \mathrm{~Hz}$ \\
Sweep frequency $\Delta f$ & $500 \mathrm{MHz}$ \\
Antenna aperture $\left(\Theta_{e l}, \Theta_{a z}\right)$ & $2^{\circ}, 2^{\circ}$ \\
Antenna rotation velocity $V_{a}$ & $120 \mathrm{rpm}$ \\
\hline
\end{tabular}

The measured radar signal is given in Figure 9(a), considering one antenna pointing direction. The signal-to-noise ratio (SNR) is $30 \mathrm{~dB}$. Figure 9 (b) shows the computed FFT radar spectrum: the spectrum highlights two main peaks, the first one corresponding to the top of the building (smallest distance) and the second one to the ground (greatest distance). Target detection is then realized in order to separate the targets echoes from the background noise. The simplest way to achieve this detection is to use a fixed threshold, but the choice of the threshold level is a complex operation. On one hand, if the level is too low, the number of detected targets will increase, as well as the number of false detections (ghost targets, or false positive). On the other hand, if the level is too high, the number of detected targets will decrease, while at the same time the risk of not detecting a target will increase (false negative). In that case, it is necessary to select an adaptive solution: the threshold level is 
automatically increased or reduced in order to maintain a constant probability of false alarm. In the radar domain, this approach is known as Constant False Alarm Rate (CFAR) detection [43],[44]. For example in a cell-averaging CFAR (CA-CFAR) approach, a local noise level is computed around each sample of the radar spectrum, and a sample will be considered as a target if its amplitude is greater than the local noise level. Figure 8(b) shows the result of such a CA-CFAR thresholding: two targets are detected, the first one (top of the building) $36.4 \mathrm{~m}$ far from the radar, the second one (ground) $44.0 \mathrm{~m}$ far from the radar.

(a)

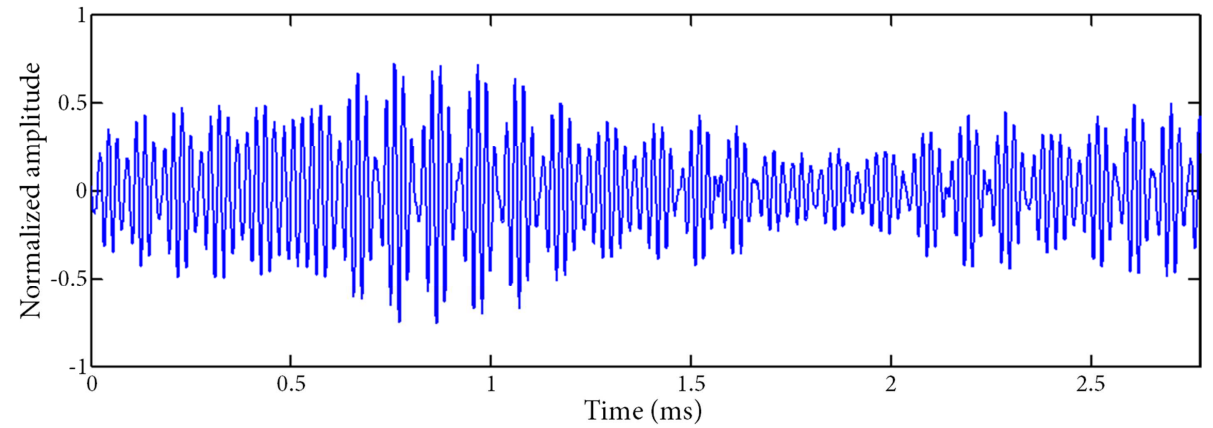

(b)

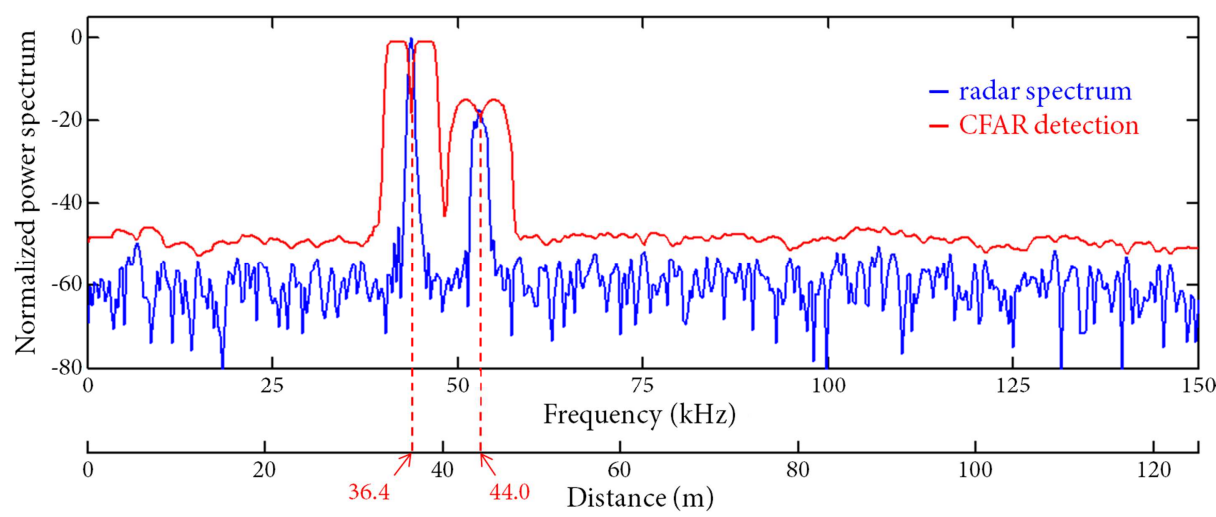

FIGURE 9: FMCW radar signal processing and target detection. (a) Temporal beat signal $s$. (b) Radar spectrum (blue line) computed with a 1024-points FFT. Two main targets are detected with the CA-CFAR detection (red line): the first one $36.4 \mathrm{~m}$ from the radar, the second one $44.0 \mathrm{~m}$ from the radar.

When considering clutter echoes (i.e. echoes from distributed targets), the signal measured by the radar is the sum of the signals backscattered by the elementary reflectors present in the radar footprint. Due to the phase term $\Phi$ in (1), this sum highlights constructive and destructive interferences. If the signals received from each reflector combine constructively (respectively destructively), the resulting measured signal will highlight a high power (respectively low power) level. Finally, the received power varies in a random fashion. With the construction of radar images, these random amplitude variations produce a speckle pattern, which is a manifestation of fading statistics. This phenomenon, called Speckle effect (or fading effect), can span several orders of magnitude, depending on the material of the targets, and on the angle of the incident wave. An illustration is presented in Figure 10. The modeled environment is a flat surface (see Figure 10(a)). Two separate areas with different surface scatter effectiveness $(-28 \mathrm{~dB}$ and $-25 \mathrm{~dB})$ are defined, and mark $P$ indicates a point target positioned on the ground. Radar parameters are described in Table 2 . The UAV follows a straight and horizontal trajectory (blue line) at the altitude of $50 \mathrm{~m}$. The incidence angle $\alpha=45^{\circ}$, and the scanning angle $\beta$ varies from $-45^{\circ}$ to $+45^{\circ}$. The measured reflected power is presented in Figure 10(b). The variations of reflected power introduced by the variations of surface scatter effectiveness allow the differentiation of both surfaces. The point target is clearly visible with a higher reflected power. The grainy salt-andpepper pattern that can be observed on the ground is an illustration of the Speckle effect. 
(a)

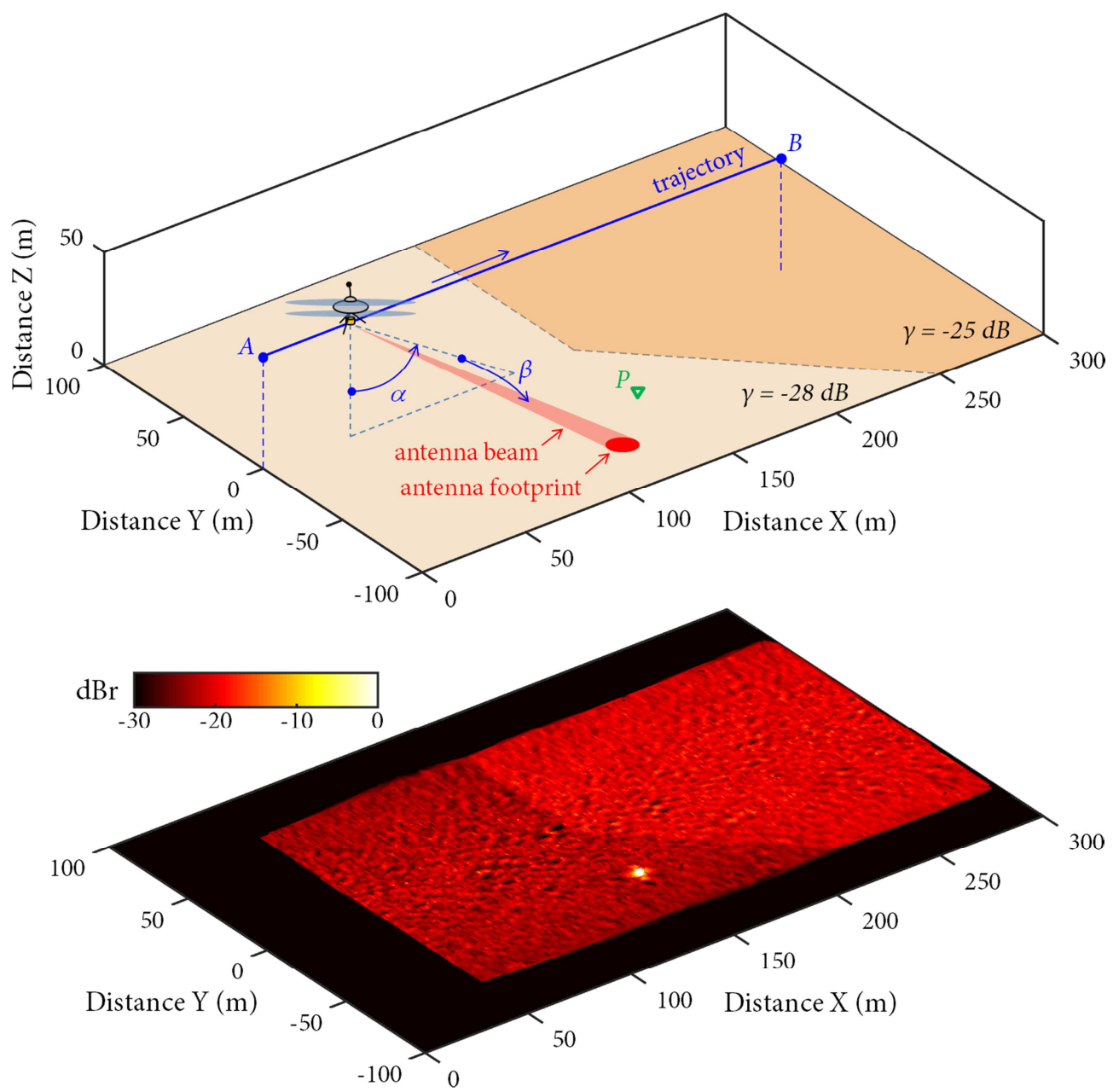

FIgURE 10: Example of radar measurement. (a) Modeled environment and UAV trajectory (blue line). Mark $P$ indicates the position of a point target on the ground. (b) Resulting measured reflected power. The variations of surface scatter effectiveness introduce variation of reflected power. The point target $P$ appears as a hotspot.

In summary, several pieces of information are measured or computed for each computation step: position and attitude of the UAV, scanning and incidence angles of the antenna, and the distances between the radar and the detected targets. By combining all these data, the positions of the detected targets are projected in a common 3D reference frame and a DEM is computed by interpolation of the detected points.

\subsection{Correction of Doppler Shift}

Considering (2), one can see that the beat frequency $f_{b}$ obtained with FMCW radar using a sawtooth modulation function depends simultaneously on radar-target distance $r$ and on radial velocity $V_{r}$. (2) highlights a range-velocity ambiguity, and without a priori knowledge on the distance or on the velocity the measurement of $f_{b}$ does not allow an unambiguous calculation of $r$ and $V_{r}$. The radial velocity between radar and target is a combination of two factors: the velocity of the target (in the case of a moving target) and the velocity of the radar itself. Within the project, the problem of moving targets is not addressed, and we assume a static environment. In that case, the radial velocity $V_{r}$ only depends on the UAV velocity $V_{\text {uav }}$ and on the angle $\vartheta$ between the direction of the UAV and the antenna direction of propagation (defined by the incidence and scanning angles, expressed in the body axis system of the UAV). An illustration is shown in Figure 11(a). The Doppler shift $f_{\text {dop }}$ can then be expressed as: 


$$
f_{\text {dop }}=\frac{2 f_{0}}{c} \underbrace{V_{u a v} \cos (\vartheta)}_{V_{r}} .
$$

A simulation of this Doppler shift is presented in Figure $11(\mathrm{~b})$, considering a carrier frequency $f_{0}=77 \mathrm{GHz}$, an UAV velocity $v_{\text {uav }}$ between -20 and $+20 \mathrm{~m} / \mathrm{s}$ and several angles $\vartheta$. For example, a velocity $v_{\text {uav }}=+14 \mathrm{~m} / \mathrm{s}$ and an angle $\vartheta=30^{\circ}$ lead to a Doppler shit $f_{\text {dop }}$ of about $6.2 \mathrm{kHz}$ : with the radar parameters described in Table 2 , the corresponding shift in distance is equal to $+5.2 \mathrm{~m}$.

(a)
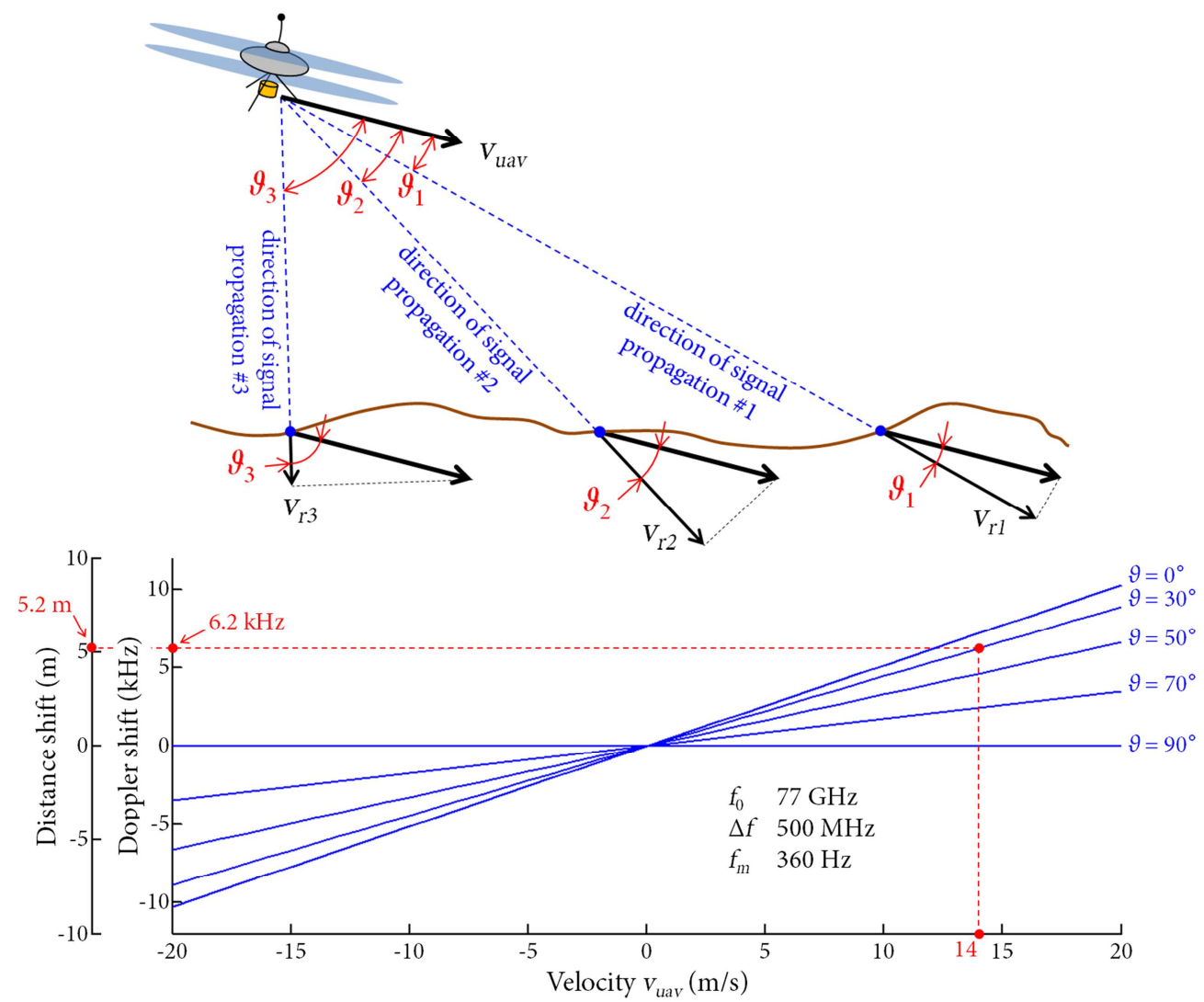

FIGURE 11: Simulation of the Doppler effect considering a static environment. (a) 2D illustration of the evolution of the radial velocity $V_{r}$ as a function of the angle $\vartheta$. (b) Variation of the Doppler shift and of the corresponding shift in distance as a function of the UAV velocity $v_{u a r}$, and different angles $\vartheta$.

In order to correct the Doppler shift, it is necessary to measure the UAV velocity $V_{u a v}$ by the use of an external sensor. By combining $V_{u a v}$ with the angular position of the radar antenna (in the UAV body axis system), one can estimate the radial velocity $v_{r}$ in the direction of signal propagation and thus the Doppler shift $f_{\text {dop }}$. Once $f_{\text {dop }}$ is estimated, the radar spectrum is shifted up or down (depending on the sign of $f_{\text {dop }}$ ) in order to compute the correct radar-target distance $r$. For example, the UAV velocity can be measured with the on-board GPS system. Considering the experience acquired by our laboratory in GPS system, a precision of $\pm 0.05 \mathrm{~m} / \mathrm{s}$ is a consistent value. With radar parameters described in Table 2 and an angle $\mathcal{\vartheta}=0^{\circ}$, we obtain a precision of $\pm 26 \mathrm{~Hz}$ for the measurement of $f_{\text {dop }}$ and a corresponding precision of $\pm 2.1 \mathrm{~cm}$ for the distance measurement $\left( \pm 18 \mathrm{~Hz}\right.$ and $\pm 1.5 \mathrm{~cm}$ with $\left.\mathcal{\vartheta}=45^{\circ}\right)$.

\section{Examples of DEM Construction}

An example of radar survey simulation is presented in Figure 12 considering a static environment. The radar parameters are those presented in Table 2. Positions and attitudes of the UAV are assumed to be known with certainty in order to focus on the influence of radar parameters. 
Figure 12(a) shows the modeled environment. The UAV follows a straight and horizontal trajectory (blue line) at the altitude of $50 \mathrm{~m}$, with a constant velocity $v_{u a v}=+10 \mathrm{~m} / \mathrm{s}$. The incidence angle $\alpha=45^{\circ}$, and the scanning angle $\beta$ varies from $-50^{\circ}$ to $+50^{\circ}$. The green points in Figure 12(b) localize the detected targets: these points are projected in a common reference frame using the computed radartargets distances, and the UAV positions and attitudes. The Doppler shift introduced by the displacement of the UAV is corrected assuming that $V_{\text {uaV }}$ is known (static environment assumption). The surface of the reconstructed DEM is obtained by interpolation of the detected targets. Due to the geometrical configuration of the radar signal acquisition, one can observe shadowing effects in the image (marks $A, B$ and $C$ for example).

Figure 12(c) is the differential DEM computed from the input data (Figure 12(a)) and the reconstructed DEM (Figure 12(b)). The global RMS error in elevation is $0.29 \mathrm{~m}$ in this example. This error is introduced by the size of the antenna beamwidth as illustrated in Figure 13(a). Because the azimuth and elevation positions of a target within the antenna beam are unknown information, the 3D reconstruction process assumes that each detected target is positioned on the central axis of the beam: in Figure 13(a), point A is the theoretical point to be detected. But all signals reflected from the $N$ scatterers present within the radar footprint are summed to produce the final beat signal $s_{b}$. Considering $N$ radar-scatterers distances between $d_{\min }$ and $d_{\max }$, the distance computed with $s_{b}$ will highlight a value $d$ ranging between $d_{\min }$ and $d_{\max }$, depending on the result of the coherent sum of $N$ elementary signals. And the distance error $e$ between the theoretical point $\mathrm{A}$ and the detected point $\mathrm{B}$ introduces a $3 \mathrm{D}$ position error during the $3 \mathrm{D}$ reconstruction process.

The simulator is an efficient tool to estimate the evolution of the RMS error in elevation as a function of the antenna aperture. The errors obtained for several antenna apertures (with the environment and configuration used in Figure 12) are presented in Table 3: it can be seen that the smaller the antenna aperture, the smaller the elevation error.

TABLE 3: Evolution of the elevation error as a function of the antenna aperture considering the environment and configuration described in Figure 12.

\begin{tabular}{crrrrl}
\hline $\begin{array}{c}\text { Antenna aperture } \\
\Theta_{a z}=\Theta_{e l}\left({ }^{\circ}\right)\end{array}$ & $\min$ & $\max$ & std & mean & RMS \\
\hline 1 & -1.24 & 1.04 & 0.18 & -0.12 & 0.22 \\
\hline 2 & -2.65 & 2.24 & 0.25 & -0.15 & 0.29 \\
\hline 4 & -6.52 & 5.19 & 0.64 & -0.24 & 0.69 \\
\hline 8 & -16.28 & 10.73 & 1.93 & -0.66 & 2.04 \\
\hline
\end{tabular}

The antenna aperture can be the source of important error in particular situations such as the one described in Figure 13(b) when the antenna beam intercepts at the same time the top of the building and the ground. In this example, the detected point $A$ is located on the top of the building. But the 3D reconstruction process assumes each detected target to be positioned on the central axis of the beam: the detected point is finally located at position $B$. The differential DEM highlights the error $h$. between point $B$ and the corresponding ground truth point.

This particular problem is simulated in Figure 14 (a), with several buildings positioned on a flat ground. Radar parameters are given in Table 2, and the radar follows a trajectory (blue line) with a constant velocity $V_{u a v}=+10 \mathrm{~m} / \mathrm{s}$ and a constant altitude of $50 \mathrm{~m}$. Figure 14 (b) is the reconstructed DEM. One can observe the shadowing effects behind the building (marks $A$ and $B$ for example), introduced by the angle of incidence $\alpha$. The differential DEM is shown in Figure 14(c). The overall RMS error in elevation is $1.51 \mathrm{~m}$ is this example, but it can be seen that the largest errors are located on the edges of the buildings (indicated with black squares). If the points near edges of buildings are 
excluded, the RMS error is only $0.19 \mathrm{~m}$. The large color gradients such as mark $C$ are artifacts introduced by the interpolation process used to display the error surface.

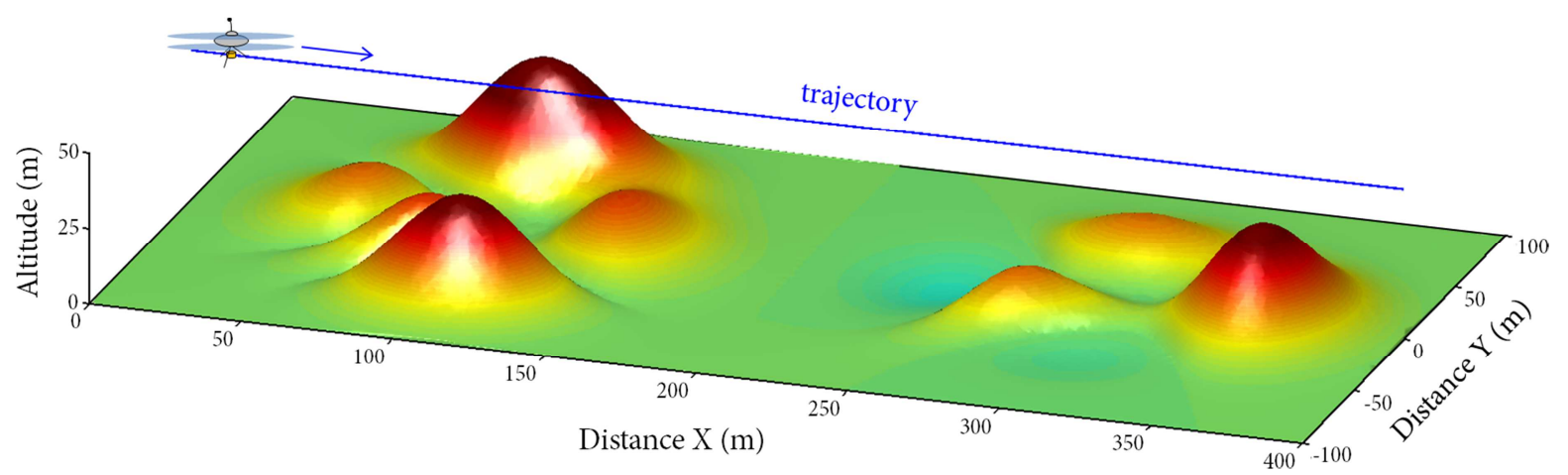

(a)

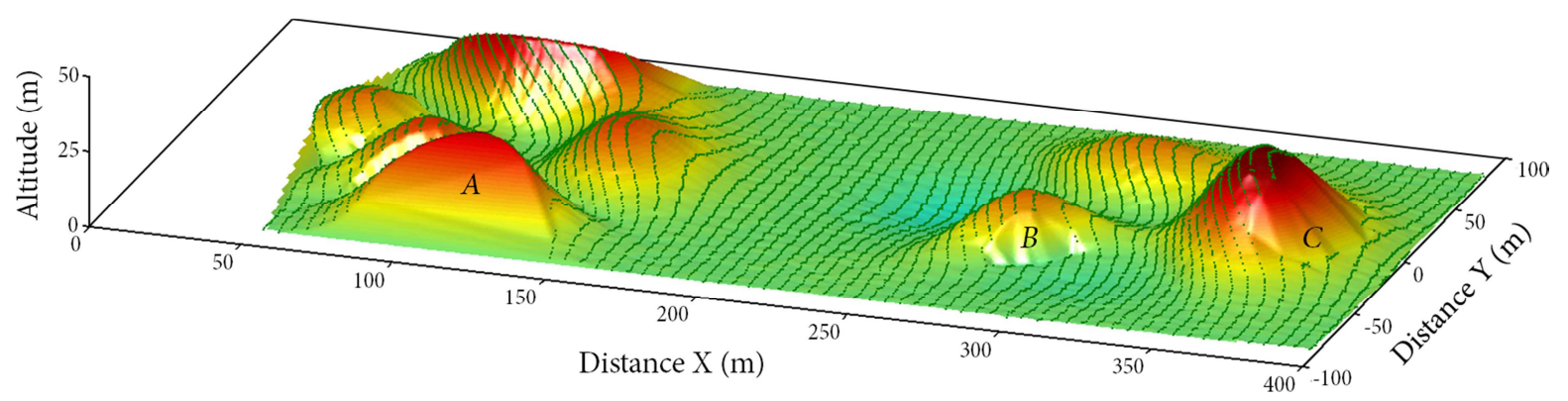

(b)

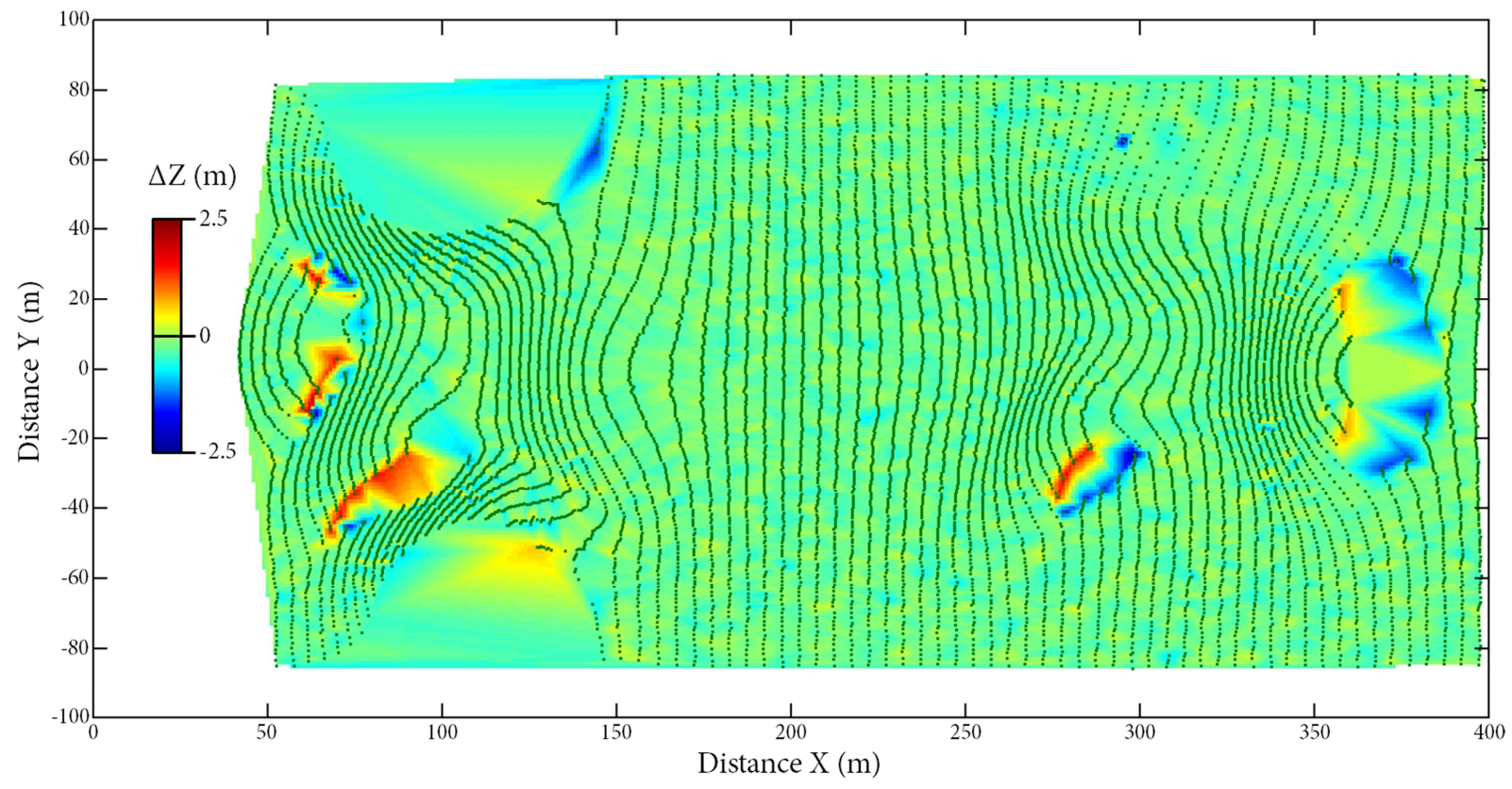

(c)

FIGURE 12: Example of radar survey simulation. (a) Model of the environment and UAV trajectory. (b) Reconstructed DEM. The green points show the detected targets for each radar acquisition. Marks $A, B$ and $C$ indicate radar shadowing. (c) Differential DEM. The overall RMS error is equal to $0.29 \mathrm{~m}$. 


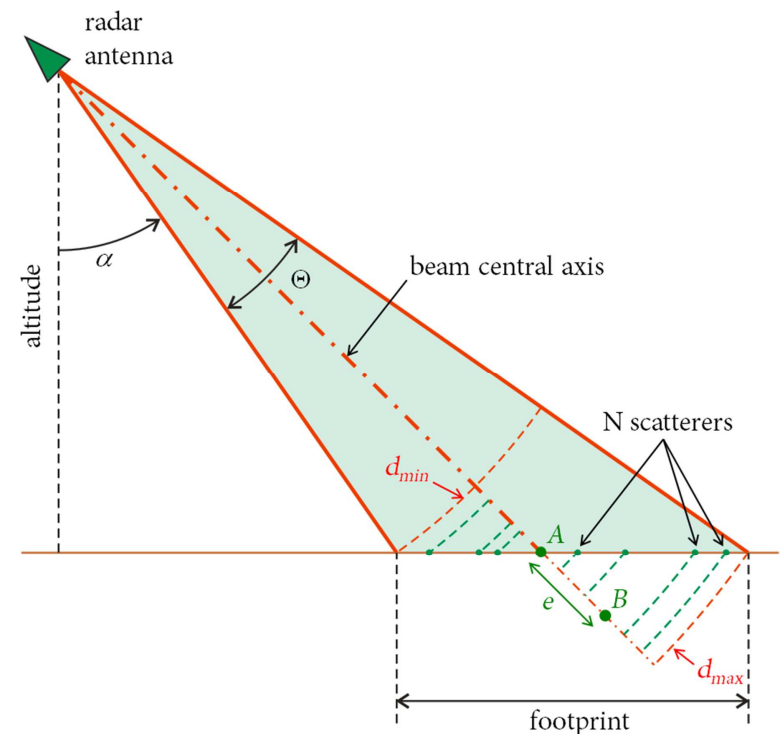

(a)

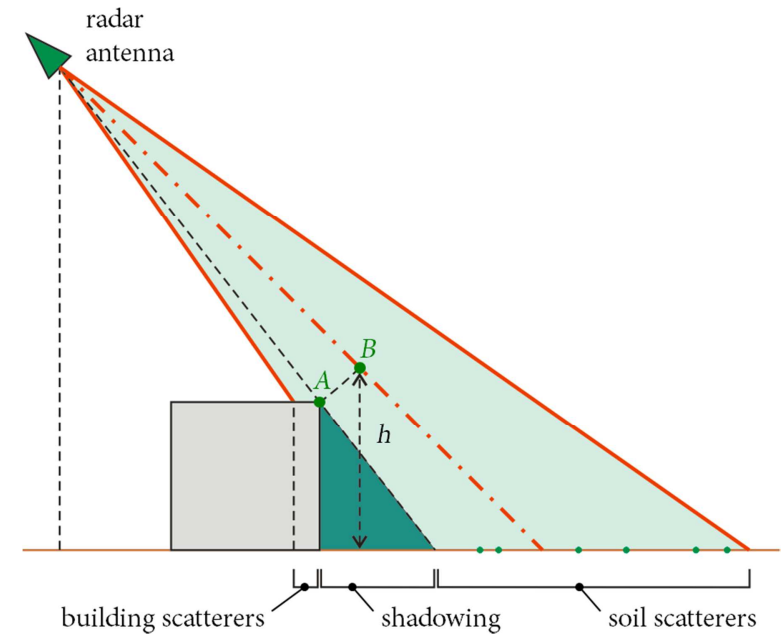

(b)

FIGURE 13: 2D illustration of the effect of antenna beamwidth on radar measurements. (a) $A$ is the theoretical point to be detected, $B$ the detected point obtained with the sum of the $N$ elementary scatterers. (b) The presence of high elements can introduce larger errors. The detected point $A$ is projected in position $B$, introducing an elevation deviation $h$ with the corresponding ground truth point.

\section{Conclusion}

For several situations, UAV are advantageous platforms for environment mapping, fulfilling the gap between satellite/aerial remote sensing and ground surveying systems. Our project is to develop a new radar sensor for UAV able to build DEM of the overflown environments, even in degraded visual conditions. A simulator is developed in order to assist the designer in defining the most efficient radar configuration for $3 \mathrm{D}$ reconstruction of the environment, with the final objective of radar sensor development.

The simulator is based on three modules: radar modeling, environment modeling, and trajectory modeling. The radar model is based on FMCW principle: this technology is well matched for short range applications, and the relative simplicity of FMCW architectures will allow the development of compact radar compatible with UAV payload. During the simulation of radar surveys, radar signals are computed and used to build the DEM of the overflown environment. The user can test a wide range of FMCW radar parameters in various environment/trajectory contexts. The objectives of the simulations are (i) to determine the optimal radar configuration in terms of distance resolution, antenna resolution (i.e. angular resolution), or antenna scanning characteristics (velocity, etc.); (ii) to fix the minimal performances of the GPS/IMU integrated navigation system which will be embedded on the UAV in order to estimate positions and attitudes; (iii) to develop the signal processing and 3D reconstruction algorithms. The conclusions will be weighted with technological constraints (technological impossibility to obtain the desired characteristics, limitations in terms of availability, size or weight incompatibility), cost constraints or current regulatory constraints (prohibited frequency bands, bandwidth and power limitations, etc.).

Beyond the specific problem addressed in the paper (DEM generation with a pencil beam radar), the simulator can be used for other purposes. It is possible to model various types of antenna with various scanning geometry, so different kind of radar can be simulated such as side-looking airborne radar (SLAR) or synthetic aperture radar (SAR). Due to a modular design, new sensor models (vision, Lidar, etc.) can be introduced, allowing work on multisensor data fusion applications. Moreover, 
considering that the radar sensor is developed, the simulator could also be used to plan the radar surveys by defining the best parameters of the UAV's flight plan.

(a)

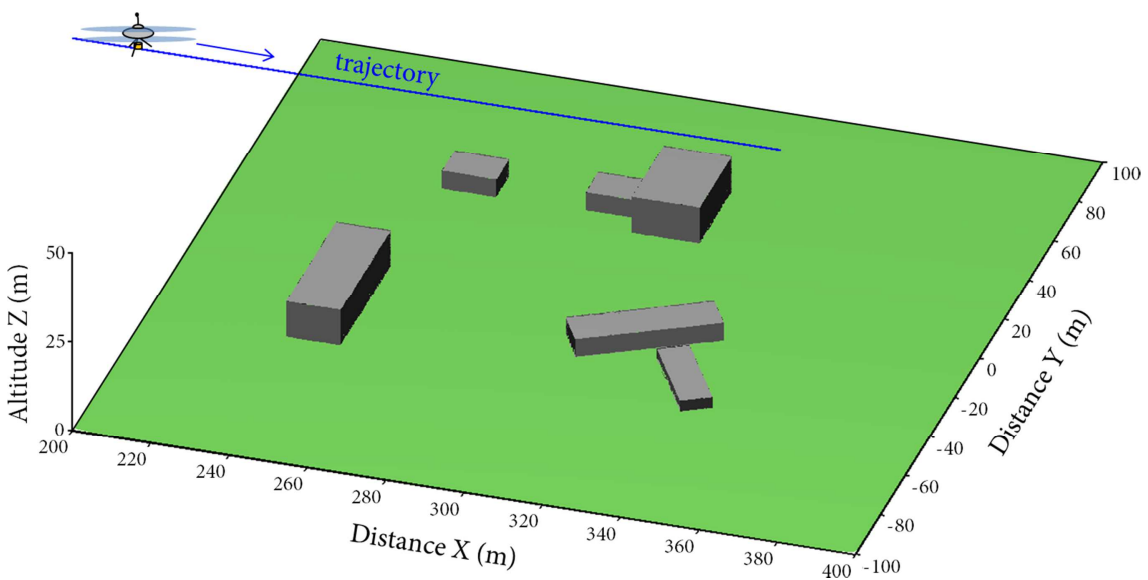

(b)

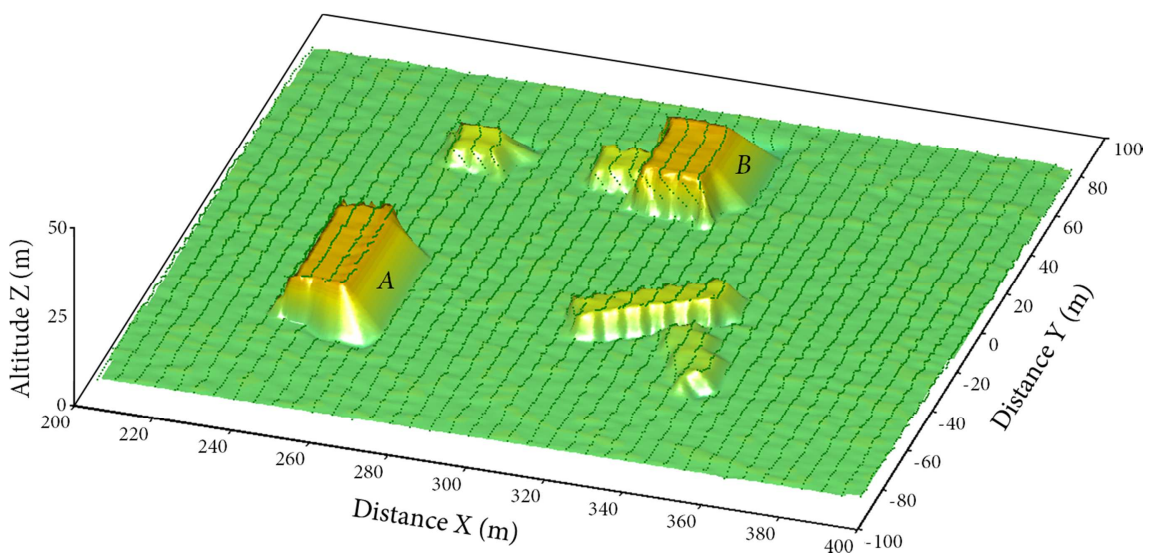

(c)

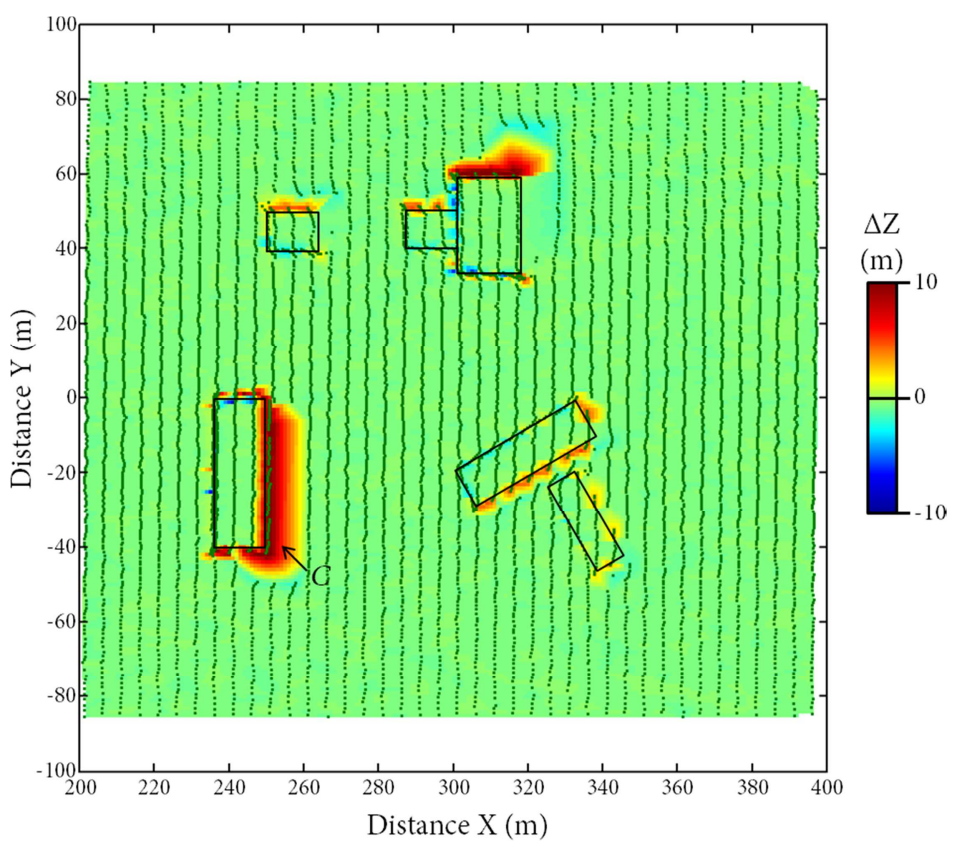

FIGURE 14: Example of radar survey simulation. (a) Model of the environment and UAV trajectory (blue line). (b) Reconstructed DEM. The green points show the detected targets for each radar acquisition. Marks $A$ and $B$ are radar shadowing. (c) Differential DEM. The largest errors are introduced by the edges of the buildings (identified with black squares). 


\section{Acknowledgments}

This work is supported by the French government research program "Investissements d'avenir" through the IMobS ${ }^{3}$ Laboratory of Excellence (ANR-10-LABX-16-01).

\section{Conflict of Interest}

The authors declare that there is no conflict of interest regarding the publication of this paper.

\section{References}

[1] D. Jenkins, and B. Vasigh, The economic impact of unmanned aircraft systems integration in the United States, Economic Report for the Association for Unmanned Vehicle Systems International, March 2013.

[2] S. Villaret, Dones civils grand public et commerciaux, potentiels et enjeux en Europe et aux Etats-Unis, IDATE Research, April 2015.

[3] L. Harriman, and J. Muhlhausen, A new eye in the sky: Eco-drones, Thematic Focus for the United Nations Environment Programme, May 2013.

[4] M.P.G. Castro, and T. Peynot, "Laser-to-Radar Sensing Redundancy for Resilient Perception in Adverse Environmental Conditions," in Proceedings of Australasian Conference on Robotics and Automation, 8 p., Wellington, New Zealand, 3-5 December 2012.

[5] G. Brooker, R. Hennessy, C. Lobsey, M. Bishop, and E. Widzyk-Capehart, "Seeing through Dust and Water Vapor: Millimeter Wave Radar Sensors for Mining Applications," Journal of Field Robotics, vol. 24, no. 7, pp. 527-557, 2007.

[6] A. Foessel, S. Chheda, and D. Apostolopoulos, "Short-Range Millimeter-Wave Radar Perception in a Polar Environment," in Proceedings of the International Conference on Field and Service Robotics, 6 p., Pittsburgh PA, USA, 29-31 August 1999.

[7] M. Caris, S. Stanko, R. Sommer, A. Wahlen, A. Leuther, A. Tessmann, M. Malanowski, P. Samczynski, K. Kulpa, M. Cohen, P. Kovacs, A. Papanastasiou, C. Topping, G. Georgiou, and R. Guraly, "SARape Synthetic aperture radar for all weather penetrating UAV application," in Proceedings of the $14^{\text {th }}$ International Radar Symposium (IRS), pp. 41-46, Dresden, Germany, 19-20 June 2013.

[8] V.C. Koo, Y.K. Chan, V. Gobi, M.Y. Chua, C.H. Lim, C.S. Lim, C.C. Thum, T.S. Lim, Z. bin Ahmad, K.A. Mahmood, M.H. Bin Shahid, C.Y. Ang, W.Q. Tan, P.N. Tan, K.S. Yee, W.G. Cheaw, H.S. Boey, A.L. Choo, and B.C. Sew, "A New Unmanned Aerial Vehicle Synthetic Aperture Radar for Environmental Monitoring," Progress In Electromagnetics Research, vol. 122, pp. 245-268, 2012.

[9] L. Wallace, "Assessing the stability of canopy maps produced from UAV-LiDAR data," in Proceedings of the IEEE International Geoscience and Remote Sensing Symposium (IGARSS), pp.3879-3882, Melbourne, Australia, 21-26 July 2013.

[10] Rouveure R, Faure P., Monod M.O., "PELICAN: Panoramic millimeter-wave radar for perception in mobile robotics applications, Part 1: Principles of FMCW radar and of 2D image construction," Robotics and Autonomous Systems, vol. 81, pp. 1-16, 2016.

[11] M.O. Monod, Frequency modulated radar: a new sensor for natural environment and mobile robotics, Ph.D. Thesis, Paris VI University, France, 1995.

[12] R. Rouveure, M.O. Monod and P. Faure, "Multiple targets detection with a FMCW radar dedicated to mobile robotics," in Proceedings of the IEEE International Radar Conference (RADAR), 3 p., Toulouse, France, 19-21 October 2004.

[13] R. Rouveure, M.O. Monod and P. Faure, "High resolution mapping of the environment with a groundbased radar imager," in Proceedings of the IEEE International Radar Conference (RADAR), 6 p., Bordeaux, France, 12-16 October 2009.

[14] M.O. Monod, R. Chapuis, P. Gosset, R. Rouveure, D. Vivet, F. Gérossier, P. Faure, P. Checchin, L. Moiroux, P. Guérin, T. Humbert, and J. Morillon, "IMPALA project. Hyperfrequency radar for outdoor simultaneous localization and mapping," Traitement du Signal, vol. 29, no. 6, pp. 463-492, 2012. 
[15] M. Jaud, R. Rouveure, L. Moiroux-Arvis, P. Faure, and M.O. Monod, "Boat borne radar mapping versus aerial photogrammetry and mobile laser scanning applied to river gorge monitoring," Open Journal of Remote Sensing and Positioning, vol. 1, pp. 48-63, 2014.

[16] S. Paquerault, H. Maitre, and J.M. Nicolas, "Radarclinometry for ERS-1 data mapping," in Proceedings of the IEEE International Geoscience and Remote Sensing Symposium (IGARSS), vol. 1. pp. 503-505, Lincoln, USA, 27-31 May 1996.

[17] D.L. Schuler, J.S. Lee, and G. De Grandi, "Measurement of topography using polarimetric SAR images," IEEE Transactions on Geoscience and Remote Sensing, vol. 34, no. 5, pp. 1266-1277, 1996.

[18] D. Massonnet, and T. Rabaute, "Radar inteferometry: limits and potential," IEEE Transactions on Geoscience and Remote Sensing, vol. 31, no. 2, pages 455-464, 1993.

[19] F. Fayard, DEM generation of a montainous area by radargrammetry and multi-windowed approach, Ph.D. Thesis, Thèse INSA Rennes, Université Européenne de Bretagne, France, 2010.

[20] D. Masters, R.S. Nerem, C. Choe, E. Leuliette, B. Beckley, N. White, and M. Ablain, "Comparison of Global Mean Sea Level Time Series from TOPEX/Poseidon, Jason-1, and Jason-2," Marine Geodesy, vol. 35, pp. 20-41, 2012.

[21] A.C. Brenner, J.P. DiMarzio, and H.J. Zwally, "Precision and Accuracy of Satellite Radar and Laser Altimeter Data Over the Continental Ice Sheets," IEEE Transactions on Geoscience and Remote Sensing, vol. 45, no. 2, pp. 321-331, 2007.

[22] B. Sykora, "BAE systems brownout landing aid system technology (BLAST) system overview and flight test results," in Proceedings of SPIE, Airborne Intelligence, Surveillance, Reconnaissance (ISR) Systems and Applications IX, vol. 8360, 15 p., Baltimore, USA, 2012.

[23] J. Cross, J. Schneider, and P. Criani, "MMW radar enhanced vision systems: the Helicopter Autonomous Landing System (HALS) and Radar-Enhanced Vision System (REVS) are rotary and fixed wing enhanced flight vision systems that enable safe flight operations in degraded visual environments," in Proceedings of SPIE, Degraded Visual Environments: Enhanced, Synthetic, and External Vision Solutions, vol. 8737, 13 p., Baltimore, USA, 2013.

[24] A. Foessel, J. Bares, and W. Whittaker, "Three dimensional map building with MMW radar," in Proceedings of the 3rd International Conference on Field and Service Robotics, 6 p., Finland, 2001.

[25] S. Scheding, G. Brooker, R. Hennessy, M. Bishop, and A. Maclean, “Terrain imaging and perception using millimeter wave radar," in Proceedings of the Australian Conference on Robotics and Automation, pp. 6065, Auckland, Australia, 27-29 November 2002.

[26] B.C. Wang, Digital Signal Processing Techniques and Applications in Radar Image Processing, WileyInterscience, New York, USA, 2008.

[27] D. Brunner, G. Lemoine, L. Bruzzone, and H. Greidanus, "Building Height Retrieval From VHR SAR Imagery Based on an Iterative Simulation and Matching Technique," IEEE Transactions on Geoscience and Remote Sensing, vol. 31, no. 2, pp. 1487-1504, 2010.

[28] Z. Wang, L. Jiang, L. Lei, and W. Yu, "Building Height Estimation from High Resolution SAR Imagery via Model-Based Geometrical Structure Prediction," Progress In Electromagnetics Research M, vol. 41, pp. 11$24,2015$.

[29] A. Taflove and S.C. Hagness, Computational Electrodynamics: The Finite-Difference Time-Domain Method, Artech House, Norwood, Massachusetts, 2005.

[30] Onier C., Chanzy A., Chambarel A., Rouveure R., Chanet M., and Bolvin H., "Impact of Soil Structure on Microwave Volume Scattering Evaluated by a Two-Dimensional Numerical Model", IEEE Transactions on Geoscience and Remote Sensing, vol. 49, no. 1, pp. 415-425, 2011.

[31] K. Kulpa, P. Samczyński, M. Malanowski, A. Gromek, D. Gromek, W. Gwarek, B. Salski, G.Tański et al., "An Advanced SAR Simulator of Three-Dimensional Structures Combining Geometrical Optics and FullWave Electromagnetic Methods," IEEE Transactions on Geoscience and Remote Sensing, vol. 52, no. 1, pp. 776-784, 2014.

[32] D. Gromek, A. Gromek, K. Kulpa, M. Malanowski, P. Samczynski, and G. Tanski, "SAR/InSAR Raw Data Simulator using DTM scene definitions," in Proceedings of the 13th International Radar Symposium (IRS), pp. 153-156, Warszawa, Poland, 23-25 May 2012. 
[33] R. Dumont, C. Guedas, E. Thomas, F. Cellier and G. Donias, "DIONISOS. An end-to-end SAR Simulator," in Proceedings of the 8th European Conference on Synthetic Aperture Radar (EuSAR), pp. 1-4., Aachen, Germany, 2010.

[34] T. Balz and N. Haala, "Improved real-time SAR simulation in urban areas," in Proceedings of the IEEE International Geoscience and Remote Sensing Symposium (IGARSS), pp. 3631-3634, 2006.

[35] T. Balz and U. Stilla, "Hybrid GPU-Based Single- and Double-Bounce SAR Simulation," IEEE Transactions on Geoscience and Remote Sensing, vol. 47, no. 10, pp. 3519-3529, 2009.

[36] H. Hammer, T. Balz, E. Cadario, U. Soergel, U. Thoennessen and U. Stilla, "Comparison of SAR simulation concepts for the analysis of high-resolution SAR data," in Proceedings of the 7th European Conference on Synthetic Aperture Radar (EuSAR), pp. 1-4, Friedrichshafen, Germany, 2008.

[37] M.I. Skolnik, Introduction to radar systems, Electrical Engineering Series, McGraw-Hill International Editions, New-York, 2nd edition, 1980.

[38] M.O. Monod, P. Faure, and R. Rouveure, "Intertwined linear frequency modulated radar and simulator for outdoor robotics applications," in Proceedings of the IEEE International Radar Conference (RADAR), 6 p., Bordeaux, France, 12-16 October 2009.

[39] D.K. Barton, Modern radar system analysis, Artech House, 1st edition, 1988.

[40] D.K. Barton, "Land clutter models for radar design and analysis," Proceedings of the IEEE, vol. 73, no. 2, pp. 198-204, 1985.

[41] M. Long, Radar reflectivity of land and sea, Artech House, 3rd edition, 2001.

[42] F.T Ulaby. and M.C.Dobson, Handbook of radar scattering statistics for terrain, Artech House, Norwood, Massachusetts, 1989.

[43] H. Rohling, "Radar CFAR thresholding in clutter and multiple target situations," IEEE Transactions on Aerospace and Electronic systems, vol. 19, no. 4, pp. 608-621, 1983.

[44] N.N. Liu, J.W. Li, and Y.F. Cui, "A new detection algorithm based on CFAR for radar image with homogeneous background," Progress In Electromagnetics Research C, vol. 15, pp. 13-22, 2010.

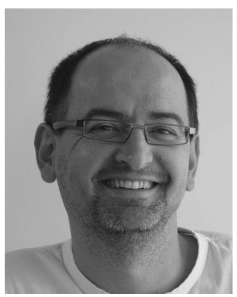

Raphaël Rouveure received the electrical engineering degree from the Polytech'Clermont School of Engineering, Clermont-Ferrand, France in 1990 and the Ph.D degree in Sciences For Engineer from the University of Clermont 2, France in 2001. He is associated with the Ecotechnologies department at the National Research Institute of Science and Technology for Environment and Agriculture (Irstea), France. His current research activities are in the development of new sensors for environmental application. His work deals with signal processing and technology in microwaves domain.

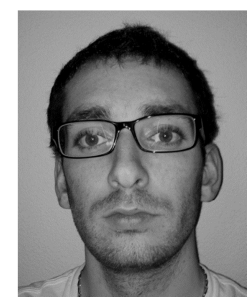

Thibault Tourrette received the electrical engineering degree from the Polytech'Clermont School of Engineering, Clermont-Ferrand, France in 2015. He is currently a Ph.D student in Sciences for Engineer from the University of Clermont 2, France. He is associated with the Technologies and Information Systems for Agrosystems (TSCF) Research Unit at the National Research Institute of Science and Technology for Environment and Agriculture (Irstea), France. His current research activities are in the development of perception algorithms and control laws for autonomous robots.

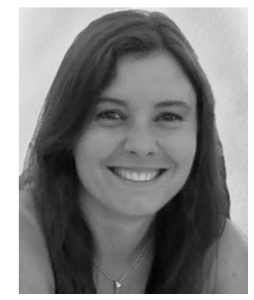

Marion Jaud received the Ph.D degree in Remote Sensing from the Bretagne Occidentale University, France in 2011. She is CNRS Research Engineer at the Oceanic Domains Laboratory (UMR 6538), European University Institute for the Sea (IUEM). Her current research activities are in the development of remote sensing methods (bathymetry, laser scanning, drone photogrammetry, FMCW radar mapping, hyperspectral imaging) for environmental monitoring. 


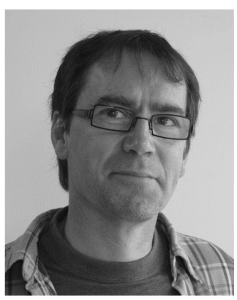

Patrice Faure received the D.E.A degree in Microelectronics from University of Montpellier, France in 1984. He is associated with the Ecotechnologies department at the National Research Institute of Science and Technology for Environment and Agriculture (Irstea), France. His research interests include the development of new sensors for environmental application. His work deals with signal processing and technology in microwaves and electronics domain.

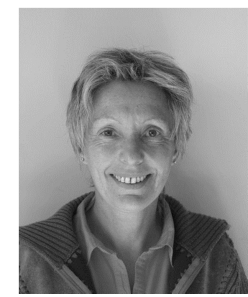

Marie-Odile Monod received the electrical engineering degree from the Ecole Supérieure d'Ingénieurs en Electronique et Electrotechnique (ESIEE Group), Paris, France in 1977 and the Ph.D degree in Physics from the University of Paris VI, Paris, France in 1995. She is associated with the Ecotechnologies department at the National Research Institute of Science and Technology for Environment and Agriculture (Irstea), France. Her research interests are in theoretical problems related to electromagnetic waves interaction with natural environment. She initiated activity in the radar sensors area for environmental perception and soil characterization. 